\title{
TOPOLOGIES OF SOCIAL INTERACTIONS
}

by

\author{
Yannis M. Ioannides ${ }^{1}$ \\ Department of Economics \\ Tufts University \\ Medford, MA 02155 \\ +16176273294_yannis.ioannides@tufts.edu
}

December 23, 2003

\begin{abstract}
The paper extends the Brock-Durlauf model of interactive discrete choice, where individuals' decisions are influenced by the decisions of others, to richer social structures. Social structure is modelled by a graph, with individuals as vertices and interaction between individuals as edges. The paper extends the mean field case to stylized interaction topologies like the Walrasian star, the cycle and the one-dimensional lattice (or path) and compares the properties of Nash equilibria when agents act on the basis of expectations over their neighbors' decisions versus actual knowledge of neighbors' decisions. It links links social interactions theory with the econometric theory of systems of simultaneous equations modelling discrete decisions. The paper obtains general results for the dynamics of adjustment towards steady states and shows that they combine spectral properties of the adjacency matrix with those associated with the nonlinearity of the reaction functions that lead to multiplicity of steady states. When all agents have the same number of neighbors the dynamics of adjustment exhibit relative persistence. Cyclical interaction is associated with endogenous and generally transient spatial oscillations that take the form of islands of conformity, but multiplicity of equilibria leads to permanent effects of initial conditions. The paper also analyzes stochastic dynamics for general interaction topologies, when agents acts with knowledge of their neighbors' actual decisions, which involve networked Markov chains in sample spaces of very high dimensionality.
\end{abstract}

JEL classification codes: C35, C45, C00, D80.

Key words: Interactions, dynamics, spatial oscillations, interactive discrete choice, neighborhood effects, Ising model, random fields, random networks.

c: Top-Soc-Inter-ASSA.tex

\footnotetext{
${ }^{1}$ I am grateful for generous research support by the National Science Foundation and the John D. and Catherine T. MacArthur Foundation. I thank Frank Alexander, Costas Azariadis, Kenneth J. Arrow, Jean-Pierre Aubin, Rob Axtell, Marcelo Bianconi, Larry Blume, Buz Brock, Marcelo Coca-Perraillon, Steven Durlauf, Glenn D. Ellison, Hans Haller, Alan Kirman, Alexandros Kyrtsis, Chuck Manski, Costas Meghir, Sharun Mukand, Lynne Pepall, Apostolis Philippopoulos, Danny T. Quah, Bertrand Roehner, Jose A. Scheinkman, Adriaan Soetevent, Dimitri Vayanos, Peyton Young, Gerard Weisbuch, Jeff Zabel and other participants in presentations of earlier versions at the joint Brookings and MacArthur Research Network on Social Interactions and Economic Inequality, the Athens University of Economics and Business, the University of Texas-Austin, ASSET, Rethymno, and the Connectionist Complexity Workshop, Institut Henri Poincaré, Paris, 2003, for comments and suggestions.
} 


\section{Contents}

1 Introduction $\quad 2$

2 Interactive Discrete Choice $\quad 4$

2.1 The Brock-Durlauf Interactive Discrete Choice Model . . . . . . . . . . . . . . 5

2.1 .1 Mean Field Theory . . . . . . . . . . . . . . . . . . . 6

2.2 Complete Interaction . . . . . . . . . . . . . . . . . . . . . . 7

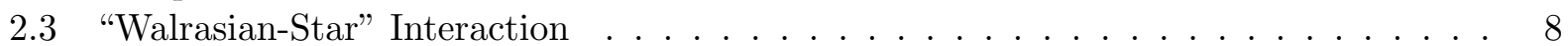

2.4 Cyclical Interaction . . . . . . . . . . . . . . . . . . . . . . 10

2.5 Path Interaction . . . . . . . . . . . . . . . . . . . . . 11

2.6 Remarks . . . . . . . . . . . . . . . . . . . . . 13

3 Interactions based on Agents' Actual Environments $\quad 13$

3.1 Cyclical Interaction as an One-Dimensional Nearest-Neighbor Ising Model . . . . . . 14

3.2 Walrasian-star Interaction . . . . . . . . . . . . . . . . . . . . 16

3.3 An Econometric Interpretation . . . . . . . . . . . . . . . . . . 17

3.3 .1 Multiplicity of Equilibria . . . . . . . . . . . . . 17

4 Dynamic Analysis of Social Interactions $\quad 18$

4.1 Stochastic Dynamics for General Topologies . . . . . . . . . . . . . . . . . . . . 19

4.1 .1 Cyclical Interaction Revisited . . . . . . . . . . . . . . . . . . . . . . 21

4.1.2 The Walrasian Star Revisited . . . . . . . . . . . . . . . . . . . 21

4.2 Dynamics with Expectations Based on Lagged Mean Decisions of Neighbors . . . . . 22

4.2.1 Dynamics of The Brock-Durlauf Mean Field Model . . . . . . . . . . . . . . . 23

4.2 .2 Dynamics of the Walrasian-Star . . . . . . . . . . . . . . . . . 24

4.2 .3 Dynamics of Cyclical Interaction . . . . . . . . . . . . . . . . . 24

4.2.4 Small versus Large Neighborhoods . . . . . . . . . . . . . . . . . 27

4.2 .5 Dynamics of Path Interaction _ . . . . . . . . . . . . . 28

4.2 .6 Dynamics with General Topologies . . . . . . . . . . . . . . . . . 29

$\begin{array}{llr}5 & \text { Extensions } & 30\end{array}$

6 Summary and Concluding Remarks $\quad 31$

$\begin{array}{lll}7 & \text { References } & 33\end{array}$

8 Appendix: Proofs and Technical Material $\quad 38$

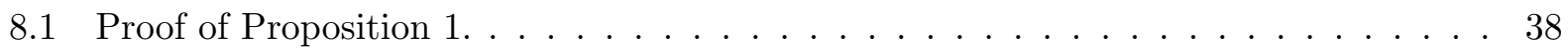

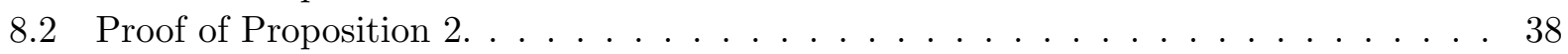

8.3 Proof of Proposition $3 \ldots \ldots \ldots \ldots \ldots \ldots$

8.4 Proof of Proposition $4 \ldots \ldots \ldots \ldots \ldots$

8.5 Proof of Proposition $5 \ldots \ldots \ldots \ldots \ldots \ldots \ldots$

8.6 Derivation of Equ. (27). . . . . . . . . . . . . . . . . . . . . 41

8.7 Derivations for the Cyclical Interaction Model as One-Dimensional Nearest Neighbor Ising Model . . . . . . . . . . . . . . . . . . . . . . . . . . . . 41

8.8 Analytics of the Dynamics of the Circular Interaction Model . . . . . . . . . . . 43

8.9 Dynamics with Path Interactions . . . . . . . . . . . . . . . . . . . 44

8.10 Dynamics with Path Interactions with $z$ Transforms when $L \rightarrow \infty \ldots \ldots$ 
8.11 Proof of Proposition $7 \ldots \ldots \ldots \ldots \ldots \ldots$ 


\section{Introduction}

This paper studies properties of economies where interdependence across agents exhibit explicit spatial interaction, which is interpreted as social interaction. It compares against the benchmark case of interaction being assumed to be uniform and global, like when each individual is assumed to be influenced by the sum of decisions of all other agents. The paper examines how the actual topology of interaction matters by comparing stylized social interaction topologies, such as where individuals are connected through a common intermediary, where the pattern of interactions forms a cycle, and where it forms an one-dimensional lattice. We show that the tools developed for the case of global interactions, which is known as the mean field case, allow us to analyze richer social interaction patterns. An important property of the dynamics of adjustment, which is shared by all regular interaction structures, that is when all agents have the same number of neighbors, is relative persistence. Some results for general interaction structures are qualitatively similar to the mean field case, but a richer class of anisotropic equilibria may arise, for the case of the cycle and onedimensional lattice topologies in static settings if the backward and forward interaction coefficients differ. Equilibria with social interactions when individuals' behavior is affected by the actual behavior of their neighbors differ qualitatively from those when individuals base their behavior on their expectations of their neighbors' decisions, both in static and dynamic settings. The paper links social interactions theory with the econometrics of systems of simultaneous equations expressing discrete decisions.

The topology of interactions has been addressed by other sciences, too. The study of dynamic spatial interaction in mathematical population biology pays special attention to the specific features of spatial interaction [ Durrett and Levin (1994) ]. Similarly, in mathematical sociology, graphtheoretic models of social interactions pay close attention to such issues [ Burt (1980); Watts and Strogatz (1998) ].

Even in economics, we often make some very specific assumptions about interaction patterns in order to obtain analytically tractable models for particular problems. These assumptions could be critically important for the properties of the economies we study. The overlapping generations model may accommodate different intertemporal interaction structures, which maps to a specific spatial structure. Cass and Yaari (1966) were the first to make this connection. For this work-horse of modern macroeconomics, economists are indeed aware of some consequences of which markets individuals have access to upon properties of competitive dynamic equilibrium in overlapping generations economies. Perhaps because intertemporal interactions in that model are mediated through markets, we might forget that market access is defined conditionally in terms of a particular interaction pattern. In fact, the originally puzzling results on the indeterminacy of equilibria, explored by Kehoe and Levine (1990) and many others, are in a sense a demonstration of the importance of the topology of interactions. Results by Puga and Venables (1997) on the consequences of patterns of preferential trading arrangements among countries again can demonstrate the same point.

Current interest in the study of direct interactions by the emerging literature on economies with 
interacting agents aims at a better understanding of conditions under which extreme, or "polarized behavior may occur solely due to collective interdependence in decision-making" [ Durlauf (1997), p. 82 ]. Durlauf also considers the coexistence of local and global interactions. Kirman (1993) emphasizes the importance of aggregation rather than summation. More recently, Young (1998) has studied coordination games that emphasize individual agent's behavior being affected by the behavior of a subset of other agents, rather than of all other agents. Kirman (1997) provides an excellent review of the overall interacting agents literature.

The possible role of an economy's interaction topology in the context of the interacting agents literature was first posed informally by Ioannides (1997). Some of the interaction topologies we examine here have been used separately by various researchers before. E.g., Ellison (1993) and Glaeser, Sacerdote and Scheinkman (1996) work with the cyclical interaction topology. Ellison considers cyclical interaction in order to work with local matching, where agents interact with a few close friends. Glaeser et al. adopt cyclical interaction because it lends itself easily to study of tractable structures when the number of agents is large. This paper is the first to bring them together under the same overarching model. Other notable contributions that underscore the importance of interaction topology are Bala and Goyal (1998), Bramoullé (2001), Haag and Lagunoff (2001), Haller and Outkin (1999) and Morris (2000). One of the contributions of the present paper is to integrate the treatment of interactions in the presence of different topologies and to identify a distict role of interaction topology in static and dynamic settings. Our results extend Brock and Durlauf (2001) in a number of ways and complement contributions by others, including in particular, Horst and Scheinkman (2003), who emphasize continuous decisions and also allow for random topologies in static models, and Bisin (2002) et al. (2002), who also emphasize continuous decisions with fixed one-sided interactions and allow for dynamics with rational expectations but exclude multiple equilibria.

In the remainder of the paper, we start first with the basic framework of interactive discrete choice and social equilibrium proposed by Brock and Durlauf (2001), which we employ as a basic building block. If an individual's behavior depends upon the sum total of the behavior of all other members of the economy, then in a Nash equilibrium setting, where each individual takes others' decisions as given and makes her own decision subject to an independent source of randomness, social equilibrium is determined as a fixed point in terms of the equilibrium value of the mean decision. The paper shows, in section 2 , that it can be extended to accommodate asymmetric interaction patterns including a Walrasian star and interaction along an one-dimensional lattice with and without closure. Section 3 examines the case where individuals' behavior is based on the actual decisions of their neighbors. We obtain the equilibrium probability distributions for the case of Walrasian-star interactions, by working from first principles, and of circular interaction, by drawing on the statistical mechanics literature on the one-dimensional Ising model. We also examine the properties of the equilibrium probability distributions for the decisions of all agents by linking interactive discrete choice models with the econometric theory of simultaneous systems of equations modelling discrete decisions. The dynamics of social equilibria are examined in Section 
4 , both for the case where individuals act with knowledge of their neighbors' actual past decisions and with expectations of their neighbors' decisions being equal to their mean past decisions. The dynamics are stochastic and thus vastly more complicated if agents use their neighbors' actual past decisions as the expectation of their future decisions. The most general case of arbitrary social interactions topology may be studied as a system of networked interactive Markov chains, but because it is defined over a very large sample sample space, its high dimensionality make it unwieldy. Nonetheless, it is associated with a stationary distribution. We characterize the stochastic dynamics in such settings for the cases when interaction topologies are translation-invariant and interaction structures reversible. These conditions are satisfied in the cases of symmetric circular interaction and of global interaction, in particular. The stochastic dynamics of the Walrasian star interaction model do not satisfy these conditions but are amenable to specific treatment. Special attention is given to the qualitative importance for the dynamics of adjustment of the social structure's exhibiting closure and regularity, that is when all individuals have the same number of neighbors. This is based on contrasting cyclical local interaction with local interaction on a path without closure. Section 5 explores possible extensions of the basic models introduced in the paper and Section 6 concludes. Proofs and technical material have been relegated to an appendix.

\section{Interactive Discrete Choice}

Let the elements of a set $\mathcal{I}$ represent individuals. Social interactions among individuals $\mathcal{I}$ are defined by an undirected graph $G(V, E)$, where: $V$ is the set of vertices, $V=\left\{v_{1}, v_{2}, \ldots, v_{I}\right\}$, an one-to-one map of the set of individuals $\mathcal{I}$ onto itself (the graph is labelled), and $I=|V|$ is the number of vertices (nodes), (known as the order of the graph); $E$ is a subset of the collection of unordered pairs of vertices and $q=|E|$ is the number of edges, (known as the size of the graph). We say that agent $i$ interacts with agent $j$ if there is an edge between nodes $i$ and $j$. Let $\nu(i)$ define the local neighborhood of agent $i: \nu(i)=\{j \in \mathcal{I} \mid j \neq i,\{i, j\} \in E\}$. The number of $i$ 's neighbors is the degree of node $i: d_{i}=|\nu(i)|$.

Graph $G(V, E)$ may be represented equivalently by its adjacency matrix, $\boldsymbol{\Gamma}$, an $I \times I$ matrix whose element $(i, j)$ is equal to 1 , if there exists an edge connecting agents $i$ and $j$, and is equal to 0 , otherwise. For undirected graphs, matrix $\boldsymbol{\Gamma}$ is symmetric and its spectral properties are important in the study of dynamics of social interactions, as we see further below. We define the diagonal matrix with $\frac{1}{\nu(i) \mid}$ as its diagonal elements as $\mathbf{N}^{-1}$.

This section develops models where agents' utility-maximizing behavior gives rises to nonlinear response rules and explores the importance of nonlinearity in conjunction with differences in the topology of social interactions for social equilibrium. We adapt the Brock- Durlauf model of interactive discrete choice that restricts attention to binary (discrete) choices [ Brock and Durlauf (2001); Durlauf (1997) ] and examine topologies of interaction other than the global one. ${ }^{2}$ By

\footnotetext{
${ }^{2}$ McKelvey and Palfrey (1995) and Chen, Friedman and Thisse (1997) develop game-theoretic discrete choice models with interactive features that are based on the logit model. However, these works do not appear to have
} 
conforming to this literature and defining the binary choice set for each individual as $S=\{-1,1\}$, we avail ourselves of the convenience of some of the analytical tools that earlier authors have already developed.

\subsection{The Brock-Durlauf Interactive Discrete Choice Model}

Let agent $i$ choose $\omega_{i}, \omega_{i} \in S$, so as to maximize her utility, which depends on the actions of her neighbors: $U_{i}=U\left(\omega_{i} ; \tilde{\omega}_{\nu(i)}\right)$, where $\tilde{\omega}_{\nu(i)}$ denotes the vector of dimension $d_{i}$ containing as elements the decisions made by each of agent $i$ 's neighbors, $j \in \nu(i)$. The $I$ vector of all agents' decisions, $\tilde{\omega}=\left(\omega_{1}, \ldots, \omega_{I}\right)$, is also known as a configuration, and $\widetilde{\omega}_{\nu(i)}$ is known as agent $i$ 's environment.

We assume that an agent's utility function $U_{i}$ is additively separable in a private utility component, which without loss of generality (due to the binary nature of the decision) may be written as $h \omega_{i}, h>0$, in a social interactions component, which is written in terms of quadratic interactions between her own decision and of the expectation of the decisions of her neighbors, $\widetilde{\omega}_{\nu(i)}$, $\omega_{i} \mathcal{E}_{i}\left\{\frac{1}{\nu(i) \mid} \sum_{j \in \nu(i)} J_{i j} \omega_{j}\right\}$, and a random utility component, $\epsilon\left(\omega_{i}\right)$, which is observable only by the individual $i$. That is, $U_{i}$ may be written as:

$$
U_{i}\left(\omega_{i} ; \mathcal{E}_{i}\left\{\tilde{\omega}_{\nu(i)}\right\}\right) \equiv h \omega_{i}+\omega_{i} \mathcal{E}_{i}\left\{\frac{1}{|\nu(i)|} \sum_{j \in \nu(i)} J_{i j} \omega_{j}\right\}+\epsilon\left(\omega_{i}\right) .
$$

The interaction coefficients may be positive - individuals are conformist - or negative - individuals are non-conformist. It will be helpful to later to define $\mathbf{J}$ as the matrix of interaction coefficients, a $I \times I$ matrix with element $J_{i j}$.

Following Brock and Durlauf (2001), in view of McFadden (1981), and under the additional assumption that $\epsilon\left(\omega_{i}\right)$ is independently and identically type I extreme-value distributed ${ }^{3}$ across all alternatives and agents $i \in \mathcal{I}$, we may write closed form expressions for the choice probabilities. For a binary choice set $S$, individual $i$ chooses $\omega_{i}=1$ with probability

$$
\operatorname{Prob}\left(\omega_{i}=1\right)=\operatorname{Prob}\left\{2 h+2 \mathcal{E}_{i}\left\{\frac{1}{|\nu(i)|} \sum_{j \in \nu(i)} J_{i j} \omega_{j}\right\} \geq-(\epsilon(1)-\epsilon(-1))\right\} .
$$

In view of the above assumptions, this may be written in terms of the logistic cumulative distribution function:

$$
\operatorname{Prob}\left(\omega_{i}=1\right)=\frac{\exp \left[\beta\left(2 h+2 \mathcal{E}_{i}\left\{\frac{1}{|\nu(i)|} \sum_{j \in \nu(i)} J_{i j} \omega_{j}\right\}\right)\right]}{1+\exp \left[\beta\left(2 h+2 \mathcal{E}_{i}\left\{\frac{1}{|\nu(i)|} \sum_{j \in \nu(i)} J_{i j} \omega_{j}\right\}\right)\right]},
$$

\footnotetext{
influenced the latest developments in this literature.

${ }^{3}$ If two independent and identically distributed random variables, $\epsilon(-1), \epsilon(1)$, obey type I extreme-value distributions, then their difference has a logistic distribution:
}

$$
\operatorname{Prob}\{\epsilon(-1)-\epsilon(1) \leq x\}=\frac{\exp [\beta x]}{1+\exp [\beta x]}
$$


where $\beta>0$ is a behavioral parameter that denotes the degree of dispersion in the random component of private utility, $\epsilon\left(\omega_{i}\right)$ in (1). The case of $\beta=0$ implies purely random choice: the two outcomes are equally likely. The higher is $\beta$ the more concentrated is the distribution. To see this, note that when $\beta$ is large, the values of $\exp [-\beta x]$ become small (large) quickly as $x$ increases (decreases), when $x>0(x<0)$. Therefore, a lot of probability is assigned to $x=0 .{ }^{4}$

The extreme-value distribution assumption for the $\epsilon$ 's is made because it yields a simple and very convenient form for the choice probabilities. It gets us a lot, by being responsible for linking with the machinery of the Gibbs distributions theory [Blume (1997); Brock and Durlauf (2001)]. ${ }^{5}$

\subsubsection{Mean Field Theory}

For the mean field theory case, considered by Brock and Durlauf (2001), if each individual's subjective expectations of other agents' decisions are equal,

$$
\mathcal{E}_{i}\left(\omega_{j}\right)=m, \forall i, j \in \mathcal{I}
$$

then the interaction term in individual $i$ 's utility function (1), and with the additional assumption that $J_{i j}=J$, becomes $\omega_{i} \frac{1}{|\nu(i)|} \sum J \mathcal{E}_{i}\left(\omega_{j}\right)=\omega_{i} J m$. Using (3) allows us to write $\mathcal{E}\left(\omega_{i}\right)$ as a function of $m$. Therefore, when individuals' subjective expectations of others' decisions are equal, $m$, then the typical individual's mean choice, $\mathcal{E}\left(\omega_{i}\right)=\operatorname{Prob}\left(\omega_{i}=1\right)-\operatorname{Prob}\left(\omega_{i}=-1\right)$, may be written from (3) as a function of his subjective expectation of the decisions by others, $m$. For Nash equilibrium, $m=\mathcal{E}\left(\omega_{i}\right)=\frac{\exp [2 \beta h+2 \beta J m]-1}{1+\exp [2 \beta h+2 \beta J m]}$, or

$$
m=\tanh (\beta h+\beta J m)
$$

where $\tanh (\cdot)$, the hyperbolic tangent function, is defined as: $\tanh (x) \equiv \frac{\exp (x)-\exp (-x)}{\exp (x)+\exp (-x)},-\infty<x<$ $\infty .^{6}$

${ }^{4}$ Translating this to the case of (3) above, we see that when $\left.\left(2 h+2 \sum_{j \in \nu(i)} J_{i j} \omega_{j}\right]\right)>0$, the term $\left.\exp \left[\beta\left(2 h+2 \mathcal{E}_{i}\left\{\sum_{j \in \nu(i)} J_{i j} \omega_{j}\right\}\right]\right)\right]$ becomes large quickly, thus increasing the probability assigned to the event $\omega_{i}=1$.

${ }^{5}$ There are, however, several additional arguments in favor of this assumption. First, the logistic integral is a fairly good approximation to the normal. Second, the extreme value distribution is the asymptotic distribution for the maximum of a set of independent random variables. That is, as $n \rightarrow \infty, Y_{n}=\max _{1 \leq i \leq n}\left\{X_{1}, \ldots, X_{n}\right\}-\ell n n$, is asymptotically extreme value distributed, where $X_{1}, \ldots, X_{n}$, are independently and identically distributed random variables with zero mean, drawn from a fairly large class of distributions [Cox and Hinkley (1974)]. This class is defined as follows. If $F(x)$ and $f(x)$ denote the probability distribution and probability density functions of the $X$ 's, and $\frac{d}{d x} \frac{1-F(x)}{f(x)} \rightarrow 0$, as $x \rightarrow \infty$, then the standardized variable $\frac{Y_{n}-a_{n}}{b_{n}}$, with $a_{n}=F^{-1}\left(1-\frac{1}{n}\right), b_{n}^{-1}=n f\left(a_{n}\right)$, has an extreme value distribution, i.e., its probability distribution function is given by $\exp [-\exp [-y]])$. This standardized extreme value distribution is skewed, with a long upper tail, and a mode at 0; its mean is equal to Euler's Constant, 0.57722 , and its variance $\frac{\pi^{2}}{6}$. [Cox and Hinkley (1974), p. 473.]

${ }^{6}$ While the hyperbolic tangent function is commonly used in statistical physics, it is rather infrequently used in economics outside the interacting agents literature. Its basic properties follow from first principles [See also Courant (1988), p. 184]. The function $y=\tanh (x)$, defined for all real values of $x$, is an increasing function with range $[-1,1]$. As $x \rightarrow-\infty(+\infty), \tanh (x) \rightarrow-1(1)$. Since $\tanh (x)^{\prime}=1-(\tanh (x))^{2}, x=0$ is both a zero and an inflection point. Therefore, the $45^{\circ}$ line through $(0,0)$ is tangent to the graph of $\tanh (x)$, leaving it above itself, for $x<0$, and below itself, for $x>0$. 
We adapt to our notation and summarize here the results of Durlauf (1997) and Brock and Durlauf (2001) regarding existence and multiplicity of the Nash equilibria given by the roots of Equ. (5). If $\beta J>1$, and $h=0$, then the function $\tanh (\beta h+\beta J m)$ is centered at $m=0$, and Equ. (5) has three roots: a positive one ("upper"), ( $\left.m_{+}^{*}\right)$, zero ("middle"), and a negative one ("lower"), $\left(m_{-}^{*}\right)$, where $m_{+}^{*}=\left|m_{-}^{*}\right|$. If $h \neq 0$ and $J>0$, then there exists a threshold $H^{*}$, which depends on $\beta$ and $J$, such that if $\beta h<H^{*}$, Equ. (5) has a unique root, which agrees with $h$ in sign. That is, given a private utility difference, if the dispersion of the random utility component is large, the random component dominates choice. If, on the other hand, $\beta h>H^{*}$, then Equ. (5) has three roots: one with the same sign as $h$, and the others of the opposite sign. That is, given a private utility difference, if the dispersion of the random utility component is small, then the social component dominates choice and is capable of producing self-consistent behavior. If $J<0$, then there is a unique equilibrium that agrees with the sign of $h$. In other words, as Durlauf underscores, Durlauf (1997), p. 88, economic fundamentals that drive private decisions and social norms play complementary roles.

We note that the model exhibits nonlinear behavior with respect to both the parameters $\beta h$, and $\beta J$. Conditional on a given private utility difference between the choices 1 and -1 , which equals $h$, there is a level which the conformity, i.e., the interaction effect must reach in order to produce multiple self-consistent mean choice behavior. However, as $\beta h$ increases in magnitude, the importance of the conformity effect $\beta J$ diminishes in a relative sense, and thus becomes unable to produce a self-consistent mean with the opposite sign. If there exist three equilibria, we will refer to the middle one $\left(m^{*}\right)$, as symmetric and to the upper and lower ones as asymmetric $\left(m_{-}^{*}, m_{+}^{*}\right)$ [ Figure 1]. Even if private incentives, expressed by $h$, favor a particular decision, sufficiently strong social conformity effects can bring about equilibria, in which individuals conform. Brock and Durlauf (2001) show that the model admits the possibility of individually optimal but collectively undesirable behavior.

\subsection{Complete Interaction}

The case of complete pairwise interaction is obtained from the general case of (1) by specifying that for each $i, \nu(i)=\mathcal{I}-\{i\}$, and $J_{i j} \equiv J_{P}, \forall j \in \mathcal{I}-\{i\}$ :

$$
U_{i}\left(\omega_{i}\right)=h \omega_{i}+\omega_{i} J_{P} \frac{1}{I-1} \mathcal{E}\left\{\sum_{\forall j \neq i} \omega_{j}\right\}+\epsilon\left(\omega_{i}\right) .
$$

Under symmetry, $\frac{1}{I-1} \mathcal{E}_{i}\left\{\sum_{\forall j \neq i} \omega_{j}\right\}=m$. Therefore, the Nash equilibrium condition is given by Equ. (5) above, with $J_{P}$ in the place of $J$. Therefore, the complete pairwise interaction case is nothing more than a way to visualize the Brock-Durlauf mean field case. We will refer to it below interchangeably as the mean field case, as well. The equilibria are obtained as the fixed points of $\tanh \left(\beta h+\beta J_{P} m\right)$. The properties of the results of the mean field case discussed above apply here 
as well. ${ }^{7}$

\section{3 "Walrasian-Star" Interaction}

This interaction model introduces a modicum of asymmetry. It is obtained from the general case above by treating agent 1 as located at the center of a "Walrasian-star", so that all others are her neighbors, $\nu(1)=\mathcal{I}-\{1\}$. All others have only agent 1 as their only neighbor: $\forall i, i \neq 1, \nu(i)=\{1\}$. We allow for a possibly asymmetric interaction intensity, by assuming $J_{1 i} \equiv J, J_{i 1} \equiv J_{S}, \forall i \neq 1$, and $J_{i j}=0$, otherwise. ${ }^{8}$ For the discrete choice model generated by (1) we have:

$$
\begin{aligned}
& U_{1}\left(\omega_{1}\right) \equiv h \omega_{1}+\omega_{1} J_{S} \frac{1}{I-1} \mathcal{E}_{1}\left\{\sum_{i=2}^{I} \omega_{i}\right\}+\epsilon\left(\omega_{1}\right) ; \\
& U_{i}\left(\omega_{i}\right) \equiv h \omega_{i}+\omega_{i} J \mathcal{E}_{i}\left\{\omega_{1}\right\}+\epsilon\left(\omega_{i}\right) ; i=2, \ldots, I .
\end{aligned}
$$

Let us assume that agents are aware of the symmetric situation of agents $i=2, \ldots, I$. They make decisions before they know the decisions of their neighbors. Individuals $2, \ldots, I$ hold common expectations of individual 1's decision, $\mathcal{E}_{i}\left\{\omega_{1}\right\}=m_{1}, i=2, \ldots, I$ and that individual 1's expectations of all others' decisions are equal: $\mathcal{E}_{1}\left\{\omega_{i}\right\}=m_{-1}, i \neq 1$. By working with the interactive discrete choice probabilities from (3) for agent 1 and agents $i=2, \ldots, n$, whose utility functions are given by (7) and (8), respectively, we may obtain conditions for social equilibrium. First, we require that the mean decision of agent 1 , as perceived by individuals outside the center, $m_{1}$, be equal to what is implied by agent 1's choice, $\mathcal{E}\left(\omega_{1}\right)=\operatorname{Prob}\left(\omega_{1}=1\right)-\operatorname{Prob}\left(\omega_{1}=-1\right)$. From (3), this may be expressed as a function of agent 1's expectation of all others' decisions, $m_{-1}$. That is:

$$
m_{1}=\tanh \left(\beta h+\beta J_{S} m_{-1}\right) .
$$

Second, individual 1's expectation of all others' decisions should be consistent with what is implied by all others' expected decisions. The latter may in turn be written in terms of all others' expectation of agent 1's decision, $m_{1}$. That is:

$$
m_{-1}=\tanh \left(\beta h+\beta J m_{1}\right) .
$$

\footnotetext{
${ }^{7}$ An interesting extension of this approach would be to assume that while every agent may interact with everyone else, a cost is involved in sampling everyone else's decisions. Therefore, the typical agent samples a finite sample of all others, and the agent's utility reflects randomness associated with this sampling. It would be interesting to investigate the dynamics of this process in view of the basic dynamics of the mean field theory model. This approach is promising, in view of the results obtained by random graph theory and of the difficulty of expressing decision-making within random graph theory models.

${ }^{8}$ Walrasian-star interaction is seen here as a prototype for trees. The model may be augmented to allow for branches with different number of nodes and may also serve as a prototype for hierarchical structures. It could represent a model for an organization where agent 1 is the principal who reacts to input by agents $2, \ldots, I$. Agent 1 , in turn, influences each of them in turn, by setting the organizational priorities. Other economic settings may be explored with this model. The extreme value distribution assumed by the behavioral model fits quite naturally a situation where agent 1 conducts an auction based on offers by agents $2, \ldots, I$. See footnote 4 . Alternatively, each of the agents on the periphery may specialize in the production of a differentiated product. The number of agents $I$ may thus reflect the demand for variety, and in fact we return to such a motivation for the model further below.
} 
The equilibria in this economy are described by the fixed points of the system of equations (9) and (10).

This case admits more possibilities than the mean field case. The equilibria are obtained as the fixed points of the mapping $\mathcal{M}$, defined from $[-1,1] \times[-1,1]$ into itself, according to Equ. (9) and (10). The results are summarized in the following proposition, whose proof is relegated to the Appendix, section 8.1. To visualize them, one could invoke a two-dimensional set of axes designated by $\left(m_{1}, m_{-1}\right)$. Then it is straightforward to draw the graphs Equ. (9) and (10), from which the solutions follow. See Figure 2. Without loss of generality, we assume that $h>0$ and recall that $\beta>0$.

Proposition 1. The system of Equations (9)-(10) admits the following roots for $\left(m_{1}, m_{-1}\right)$.

(a) If $J, J_{S}>0$, then there exist at least one root that lies in the set $(0,1) \times(0,1)$. If, in addition, $\beta J>1$, and $\beta J_{S}>1$, then there exists a threshold $H^{+}$, such that if $\beta h<H^{+}$, then there exist two additional roots that lie in $(-1,0) \times(-1,0)$.

(b) If $J>0, J_{S}<0$, then there always exist a single root, such that lie in $(-1,1) \times(0,1)$.

(c) If $J<0, J_{S}>0$, then there always exist a single root, such that lie in $(0,1) \times(-1,1)$.

(d) If $J<0, J_{S}<0$, then there exist at least one root that lies in the set $(0,1) \times(-1,0)$. If, in addition, $\beta J<-1$, and $\beta J_{S}<-1$, then there exists a threshold $H^{-}$, such that if $\beta h<H^{-}$, then there exist two additional roots that lie in $(-1,1) \times(0,1)$.

As in the mean field case, if there exist three equilibria, we refer to the "middle" one as symmetric and the other two as asymmetric. It is interesting that when both interaction coefficients have the same sign the resulting equilibria are characterized by the following properties. If both interaction coefficients are positive then there is always at least one root that is in the positive orthant of $\left(m_{1}, m_{-1}\right)$ space. That is, if agents $1, \ldots, I$, are optimistic about agent 1 , and agent 1 optimistic about agents $1, \ldots, I$, the upper equilibrium prevails: conformism is an equilibrium. If both groups of agents are pessimistic about the other group the lower equilibrium is also possible, provided that the interactions effect is sufficiently strong to overcome the private effect. If both interaction coefficients are negative then at least one root is associated with a positive solution for $m_{1}$ and a negative one for $m_{-1}$ : conformism is again an equilibrium. When, on the other hand, preferences are different, in the sense that agent 1 does not wish to conform but the agents $2, \ldots, I$ do, then agents $2, \ldots, I$ choose an expected decision in the positive orthant, $m_{-1}>0$, but agent 1 might not, $m_{1}$ may be positive or negative. If, on the other hand, agent 1 does not wish to conform but agents $2, \ldots, I$ do, agents $2, \ldots, I$ choose an expected decision in the positive orthant, $m_{1}>0$, but agent 1 might not, $m_{-1}$ may be positive or negative.

The equilibria of the Walrasian interaction model that are established by Proposition 1 allow for the possibility of qualitatively different behavior by the two groups of agents. Still, the different Nash equilibria of the economy must be understood as pairs of consistent outcomes. 


\subsection{Cyclical Interaction}

Cyclical interaction occurs when each agent interacts only with the agent to her right and to her left. That is, for each $i$, her neighborhood is defined $\nu(i)=\{i-1, i+1\}$, and $J_{i, i-1} \equiv J_{B}, J_{i, i+1} \equiv J_{F}$. For symmetry, the agent to agent I's right is agent $1,\{I+1\}=\{1\}$, and the agent to agent 1's left is agent $I,\{1-1\}=\{I\}$. Cyclical interaction has been used before, notably by Glaeser et al. (1996) and by Young (1998), p. 6-10, but in applications that are somewhat different from the present one.

From the discrete choice model generated by (1) we have by using the above restrictions:

$$
U_{i}\left(\omega_{i}\right) \equiv h \omega_{i}+\omega_{i} \frac{1}{2} \mathcal{E}_{i}\left\{J_{B} \omega_{i-1}+J_{F} \omega_{i+1}\right\}+\epsilon\left(\omega_{i}\right) .
$$

Let agent $i$ 's expectation of the decision by her neighbor to her left be $m_{i-1}$ and to her right be $m_{i+1}$. Again, individuals make decisions under expectations over the decisions of their neighbors and before finding out their neighbors' decisions. Then, for consistency, agent $i$ 's expected decision, $m_{i}$, must be equal to that implied her own decision, $\mathcal{E}\left(\omega_{i}\right)=\operatorname{Prob}\left(\omega_{i}=1\right)-\operatorname{Prob}\left(\omega_{i}=-1\right)$, which may be expressed in terms of $m_{i-1}$ and $m_{i+1}$ :

$$
m_{i}=\tanh \left[\beta h+\frac{1}{2} \beta\left(J_{B} m_{i-1}+J_{F} m_{i+1}\right)\right] .
$$

By symmetry, the above condition applies for all $i$ s. We thus have a system of $I$ equations in the $I$ unknowns $m_{i}, i=1, \ldots, I$, with "initial" conditions $m_{I+1}=m_{1}$, and $m_{I+2}=m_{2}$.

It is straightforward to show that if we impose that all agents' expected decisions are equal, then the Nash equilibria of this setting are similar to those of the mean field case. In the context of the cyclical interaction model, we refer to such equilibria as isotropic. The following question then arises. Is it possible to have as equilibria a sequence of numbers that may differ from one another and also obey circular symmetry? It turns out that this is possible only if the backward and forward interaction coefficients are different: $J_{B} \neq J_{F}$. These results are established by Proposition 2, whose proof is given in the Appendix, section 8.2. We refer to such equilibria as anisotropic. Proposition 2. The system of Equations (12), $i=1, \ldots, I$, that describe cyclical interaction defines a mapping in $R^{I}$ into itself

$$
\mathcal{C}: R^{I} \rightarrow \underbrace{(-1,1) \times \ldots(-1,1)}_{I} .
$$

It may have, in general, two classes of solutions, isotropic and anisotropic ones.

(a) There exist, in general, isotropic solutions, $m_{i}=m_{C I}^{*}, \forall i$, where $m_{C I}^{*}$ is a root of

$$
m=\tanh \left[\beta h+\frac{1}{2} \beta\left(J_{B}+J_{F}\right) m\right] .
$$

Their properties are identical to those obtained by Durlauf (1997), Theorem 1, p. 88, discussed above, with the only difference being that $\frac{1}{2}\left(J_{B}+J_{F}\right)$ takes the place of $J$ in ibid. There may be either three distinct roots, or a single root, that all lie in $(-1,1)$. 
(b) If $J_{B}=J_{F}$, then all solutions are isotropic. If $J_{B} \neq J_{F}$, then anisotropic solutions may exist and are given by the fixed points of $\Theta_{[I-2]}$, the $I-2$ th iterate of a mapping $\Theta=\left(\Theta^{1}, \Theta^{2}\right)$, defined as follows:

$$
\begin{aligned}
\Theta^{1}\left(m^{\prime}, m^{\prime \prime}\right) & \equiv \frac{1}{\frac{1}{2} \beta J_{F}}\left[\tanh ^{-1}\left(m^{\prime}\right)-\beta h\right]-\frac{J_{B}}{J_{F}} m^{\prime \prime} \\
\Theta^{2}\left(m^{\prime}, m^{\prime \prime}\right) & \equiv m^{\prime}
\end{aligned}
$$

where $\left(m^{\prime}, m^{\prime \prime}\right) \in(-1,1) \times(-1,1)$, and $\tanh ^{-1}(\cdot)$ denotes the inverse hyperbolic tangent function. (c) Anisotropic solutions may exist for the case of $I=3$ agents and are given by the fixed points of $\Theta_{[1]}$, the first iterate of $\Theta$.

(d) Anisotropic solutions do not exist in the case of either only backward interaction, $J_{F}=0$, or only forward interaction, $J_{B}=0$.

I have not been able to prove the general case for $I>3$. I note, however, that the possibility of an anisotropic solution rests on the bilateral nature of the interactions, in view of part $d$, and on the nonlinearity of the problem. ${ }^{9}$ This is demonstrated by the following result, whose proof is given in the Appendix, section 8.3.

Proposition 3. The system of Equations (12), $i=1, \ldots, I$, do not possess an anisotropic solution in the vicinity of one of its isotropic solutions.

\subsection{Path Interaction}

With the set of agents being defined as $\mathcal{I}=\{-L, \ldots, 0, \ldots, L\}$, the equilibrium conditions for agents $i=-L+1, \ldots, L-1$, are as in (12), $-L+1 \leq i \leq L-1$. For agents $-L$ and $L$, the equilibrium conditions are:

$$
\begin{aligned}
m_{-L} & =\tanh \left[\beta h+\beta J_{F} m_{-L+1}\right] ; \\
m_{L} & =\tanh \left[\beta h+\beta J_{B} m_{L-1}\right] .
\end{aligned}
$$

Equ. (12), for $i=-L+1, \ldots, L-1$, and Equ. (15) and (16), form a system of $2 L+1$ equations in the $2 L+1$ unknowns, the expected states of all agents.

This setting is similar to the cyclical interaction case, except for the presence of two end agents. To see this intuitively, consider the classic example from Schelling (1978): "If everybody needs 100 Watts to read by and a neighbor's bulb is equivalent to half one's own, and everybody has a 60-Watt bulb, everybody can read as long as he and both his neighbors have their lights on. Arranged on a circle, everybody will keep his lights on if everybody else does (and nobody will if his neighbors do not); arranged in a line, the people at the ends cannot read anyway and turn their lights off, and the whole thing unravels" [ibid. p. 214]. One would expect that the importance of the two end agents would wane as the number of agents increases and as preferences differ from the fixed-proportions case implied by the above example. Also, the above example suggests that it might be important to allow for boundary conditions, as when the end agents, or the agent at 0 , are constrained to be in a particular state.

\footnotetext{
${ }^{9}$ In that sense, the search for anisotropic solutions resembles the establishment of periodic solutions in the theory of nonlinear difference equations [ See Azariadis (1993), p. 85-88 ].
} 
We improve our intuition of interaction on a path by considering two extreme cases, one where there are just three agents, $L=1$, and another where there are an infinite number of agents. The proposition that follows treats separately the case of just three agents, $L=1$, from that of the general case. Starting with the three-agent case, $L=1$, Equ. (15) yields $m_{-1}$ as a function of $m_{0}$, and Equ. (16) yields $m_{1}$ as a function of $m_{0}$. By substituting back into the R.H.S. of (12) we obtain a single equation in $m_{0}$. Under the assumption that the interaction coefficients are both positive, we can easily see that the existence of the end agents strengthens the case for multiplicity of equilibria. Our results are summarized in the following proposition, whose proof is given in the Appendix, section 8.4.

Proposition 4. The system of Equations (12), $-(L-1) \leq i \leq L-1$, and Equations (15) and (16) that describe interaction on a path defines a mapping in $R^{I}$ into itself:

$$
\mathcal{C}: R^{I} \rightarrow \underbrace{(-1,1) \times \ldots(-1,1)}_{I} \text {. }
$$

It has two classes of solutions, isotropic and anisotropic ones.

(a) There exist in general isotropic solutions, $m_{i}=m_{L I}^{*},-(L-1) \leq i \leq L-1$, which are the roots of

$$
m=\tanh \left[\beta h+\frac{1}{2} \beta\left(J_{B}+J_{F}\right) m\right]
$$

and $m_{-L}, m_{L}$, follow from (15) and (16), as functions of $m_{L I}^{*}$. Their properties are identical to those obtained by Durlauf (1997), Theorem 1, p. 88, discussed above, with $\frac{1}{2}\left(J_{B}+J_{F}\right)$ in the place of $J$ in Equ. (5). There may be either three distinct roots, or a single root, that all lie in $(-1,1)$. (b) For $L=1$, the case of three agents, the solution for $m_{0}$ is obtained as a solution of,

$$
\tanh ^{-1}\left(m_{0}\right)=\beta h+\frac{1}{2} \beta J_{B} \tanh \left[\beta h+\beta J_{F} m_{0}\right]+\frac{1}{2} \beta J_{F} \tanh \left[\beta h+\beta J_{B} m_{0}\right]
$$

and $m_{-1}, m_{1}$, follow from (15) and (16), as functions of $m_{0}^{*}$. Equ. (18) has, depending upon parameter values, either three distinct roots, of which one has the same sign as $h$ and the other two with the opposite sign, or a single root with the same sign as $h$. Furthermore, $m_{-1}=m_{0}=m_{1}$, only if $J_{B}=J_{F}$; otherwise, they are different.

(c) The anisotropic solutions are given in terms of the solutions of

$$
\tanh ^{-1}\left(m_{0}\right)=\beta h+\frac{1}{2} \beta J_{B} \Theta_{L-1}^{+}\left(m_{0}\right)+\frac{1}{2} \beta J_{F} \Theta_{L-1}^{-}\left(m_{0}\right)
$$

where the mappings $\Theta_{L-1}^{+}(\cdot), \Theta_{L-1}^{-}(\cdot)$, are defined in an iterative fashion below.

As in the case of cyclical interaction, the possibility of an anisotropic solution rests entirely on the nonlinearity of the problem. This is demonstrated by the following result, whose proof is given in the Appendix, section 8.5.

Proposition 5. The system of Equations (12), $-(L-1) \leq i \leq L-1$, and Equations (15) and (16) that describe interaction on a path do not, in general, possess an anisotropic solution in the vicinity of one of its isotropic solutions. 
For the case of the infinite line, equilibrium interactions are described by infinite sequences $\left\{m_{i}\right\}_{-\infty}^{\infty}$ that satisfy Equ. (12). Consideration of an infinite economy attenuates the role of the end agents and prompts the following question. Does a spatial anisotropic equilibrium exist, that is, are there anisotropic sequences with $m_{i} \neq m_{i+1}$ that satisfy Equ. (12)? We have not been able to explore this formally, but it appears, intuitively, that the answer to this question is negative.

\subsection{Remarks}

Three remarks are in order. First, if we assume that all interaction coefficients in all of the above models are equal to one another, $J_{P}=J_{B}=J_{F}=J_{S}=J$, then the equilibria of all models coincide with those of the Brock-Durlauf mean field case, Equ. (5). While not surprising, as that assumption imposes symmetry, it will be useful to bear in mind this property when we discuss equilibrium with interactions that depend on agents' actual environments, in static and dynamic settings, sections 3 and 4.1, respectively.

Second, at any of the isotropic equilibria examined above, which are associated with individuals' making decisions, conditional on their expectations of their neighbors' decisions, individuals' states are described by means of independent Bernoulli distributed random variables. These are defined by the respective choice probabilities as functions of the mean values at equilibrium. A most noteworthy property that all three models share is that the social equilibrium may be characterized by aggregate uncertainty, even when individual states are purely random. That is, consider the case when $h=0$, the two states equally likely in terms of fundamentals. Then, even in the mean field case, the economy has three isotropic equilibria, associated with the roots of Equ. (5), for $h=0$. One of them is $m=0$, and indeed implies no aggregate uncertainty: the expected outcome is equal to 0 . However, this social equilibrium is unstable. If $\beta J>1$, then the other two roots imply aggregate activity, and the corresponding equilibria are stable. Naturally, the emergence of aggregate activity is due to the synergistic effects operating at the individual level.

Third, since anisotropic equilibria will be excluded by the assumptions we make in the remainder of the paper, it is important to stress that they model the consequences of a basic lack of symmetry in the economy. The islands of conformity that would appear, which become clearer below in the analysis of circular interaction when agents make decisions based on their neighbors' actual decisions, are not completely random; they are instead skewed.

\section{Interactions based on Agents' Actual Environments}

We have so far analyzed equilibria with social interactions under the assumption that each agent acts with beliefs about expected decisions of her neighbors. It should not be surprising that all social interaction structures that exhibit local symmetry, that is the complete, circular and path interactions away from the two ends, have the same Nash equilibria, when all interaction coefficients are assumed to be equal. With local interactions, where one knows one's neighbors, it is reasonable to assume that an individual observes her neighbors' actions. It is therefore important to examine 
also the impact of interaction topology on equilibria when agents know the actual decisions of their neighbors when they make their own decisions. The requirement that agents' optimal decisions be consistent with one another at equilibrium allows us, in principle, to obtain the probability distribution functions for agents' decisions in terms of fundamentals and of the distributions of the random utility components. The aspect of the model examined here is novel in the context of the interactive discrete choice literature. ${ }^{10}$

Specifically, the social component of individual $i$ 's utility is defined according to (1) but without expectations, $\omega_{i} \sum_{j \in \nu(i)} J_{i j} \omega_{j}$. Utility maximization by individual $i$, conditional on $\tilde{\omega}_{\nu(i)}$ yields a conditional version of $(3)$ :

$$
\operatorname{Prob}\left(\omega_{i}=1 \mid \tilde{\omega}_{\nu(i)}\right)=\frac{\exp \left[\beta\left(2 h+2 \frac{1}{|\nu(i)|} \sum_{j \in \nu(i)} J_{i j} \omega_{j}\right)\right]}{1+\exp \left[\beta\left(2 h+2 \frac{1}{|\nu(i)|} \sum_{j \in \nu(i)} J_{i j} \omega_{j}\right)\right]}, i=1, \ldots, I .
$$

This is a description of each agent's best response conditional on her environment. It coincides with the conditional specification of a Markov random field: the probability distribution of each agent's state depends on those of her neighbors. A key result from the literature on Markov random fields states that if this is a strictly positive nearest neighbor specification, then there exists a single probability distribution function for the state of the economy $\tilde{\omega}$, known as global phase, which is consistent with the local specification [Kindermann and Snell (1980)]. The global phase is a Markov random field, and in fact every Markov random field is equivalent to a Gibbs state for some single nearest neighbor potential [ Kindermann and Snell (1980) ].

Equilibrium with social interactions when agents know the actual decisions of their neighbors thus have very different structure than those of the Brock-Durlauf model. Being described by means of a probability distribution function that coincides with the global phase of the respective Markov random field, this distribution is characterized by more general dependence than dependence on each agent's neighbors. ${ }^{11}$ We turn next to examine in detail two specific topologies. As an indication of how they differ from the Brock-Durlauf setting, the explicit solutions for agents' equilibrium behavior that we obtain do not involve fixed points, unless agents' behavior also depends on the expected behavior of other agents because of presence of local and global interaction [cf. Horst and Scheinkman (2003), sections 3, 4].

\subsection{Cyclical Interaction as an One-Dimensional Nearest-Neighbor Ising Model}

We demonstrate the power of this theory by applying the classic treatment of Baxter (1982), p. 32-36 to deriving expressions for the marginal probabilities of agents' decisions for the model of cyclical interaction, when agents observe the decisions of their neighbors. We present and discuss here only the results and give the details of the derivations in the Appendix, section 8.7. For that

\footnotetext{
${ }^{10}$ Horst and Scheinkman (2003) examine the continuous decisions case with social interactions that depend on actual decisions of their neighbors and on the empirical distribution of actions throughout the economy.

${ }^{11}$ As Horst and Scheinkman (2003), p. 26-27, put it in their analysis of continuous decisions: "the distributions of equilibrium action profiles is not specified by the family of individual best response functions."
} 
model, the probability of a social state $\tilde{\omega}$ is given by

$$
\operatorname{Prob}(\tilde{\omega})=\frac{1}{\lambda_{1}^{I}+\lambda_{2}^{I}} \exp \left[\beta J \sum_{j=1}^{I} \omega_{j} \omega_{j+1}+\beta h \sum_{j=1}^{I} \omega_{i}\right],
$$

where $\lambda_{1}, \lambda_{2}, \lambda_{1}>\lambda_{2}$, are the two distinct real eigenvalues of a positive symmetric matrix, which is defined in the Appendix, section 8.7. It follows from the characteristic equation of this matrix that the eigenvalues are either both positive, if $J>0$, or one is positive (the absolutely larger of them) and the other is negative, if $J<0$. Therefore, the denominator in Equ. (21) is always positive.

The marginal probability for the state of each agent is:

$$
\operatorname{Prob}\left\{\omega_{i}=1\right\}=\frac{(\cos \xi)^{2} \lambda_{1}^{I}+(\sin \xi)^{2} \lambda_{2}^{I}}{\lambda_{1}^{I}+\lambda_{2}^{I}},
$$

where the auxiliary variable $\xi$ is defined implicitly by:

$$
\cot 2 \xi=e^{2 \beta J} \frac{e^{\beta h}-e^{-\beta h}}{2} \equiv e^{2 \beta J} \sinh (\beta h), 0<\xi<\frac{\pi}{2} .
$$

The marginal probability describing the state of each agent, the reduced form, is uniquely defined in terms of the fundamentals. Therefore, outcomes for agents cannot be specified independently, as functions of fundamentals, in a regression setting.

When $I \rightarrow \infty$, while $j-i$ remains finite, Baxter, op. cit. shows that

$$
\begin{gathered}
\mathcal{E}\left[\omega_{i}\right]=\cos 2 \xi \\
\mathcal{E}\left[\omega_{i} \omega_{j}\right]=(\cos 2 \xi)^{2}+\left(\frac{\lambda_{2}}{\lambda_{1}}\right)^{j-i}(\sin 2 \xi)^{2}, j \geq i,
\end{gathered}
$$

This implies that the covariance function between $\omega_{i}$ and $\omega_{j}$ is given by: $g_{i j}=\left(\frac{\lambda_{2}}{\lambda_{1}}\right)^{j-i}(\sin 2 \xi)^{2}, j \geq$ $i$. The variance of $\omega_{i}$ is equal to $(\sin 2 \xi)^{2}$. Therefore, the correlation between the decisions of agents $i$ and $j$ depends only on the distance between them, $j-i$, and declines as that increases because $\lambda_{1}>\lambda_{2}$.

The larger is $J$, the social interaction coefficient, the smaller is $\xi$, the larger is the mean state of the typical agent, the smaller its variance, and the smaller its correlation with other agents' states. Therefore, the more important are social interactions, the larger and less diverse is aggregate activity. It is also interesting to note that if the social interaction coefficient is negative, $J<0$, that is individuals are nonconformist, then the correlation coefficient between the states of any two agents alternates between being negative for adjacent agents and then positive for agents two sites apart, and so on. We may interpret this as clustering. Non-conformism leads to "islands" of individuals who are more likely to be in similar states, the closer they are to one another, and the states of these islands alternate.

Although existence of a social equilibrium through existence of a global phase is guaranteed, it is still interesting to compute the equilibrium probabilities associated with the different social structures when individuals make decisions, conditional on their actual environments. Additional intuition is gained by working from first principles and derive marginal probabilities for agents' decisions in the context of the Walrasian-star and cyclical interaction topologies. 


\subsection{Walrasian-star Interaction}

We consider utilities being given by (7) and (8), except that instead of the expectations over the neighbors' states we have that utilities depend on the actual realizations, that is neighbors' actual decisions. The agents are assumed to make contingent plans, that is contingent on their neighbors' decisions. The plans imply choice probabilities as follows:

$$
\operatorname{Prob}\left\{\omega_{1}=1 \mid \sum_{j=2}^{I} \omega_{j}\right\}=\frac{\exp \left[2 \beta h+2 \frac{1}{I-1} \beta J_{S} \sum_{j=2}^{I} \omega_{j}\right]}{1+\exp \left[2 \beta h+2 \frac{1}{I-1} \beta J_{S} \sum_{j} \omega_{j}\right]},
$$

and

$$
\operatorname{Prob}\left\{\omega_{i} \mid \omega_{1}\right\}=\frac{\exp \left[2 \beta h+2 \beta J \omega_{1}\right]}{1+\exp \left[2 \beta h+2 \beta J \omega_{1}\right]} ; i=2, \ldots, I .
$$

Imposing the condition that agents' plans are mutually consistent allows us to compute the marginal probabilities of agents' states.

From (26) we note that the states of agents $\omega_{i}, i=2, I$, are conditionally independent. Therefore, the random variable $\sum_{j=2}^{I} \omega_{j} \mid \omega_{1}$ may be studied in terms of the binomial distribution, the probability of the number of successes in $I-1$ Bernoulli trials, conditional on $\omega_{1}$. Since any $\omega_{i}$ may assume the value of either 1 or -1 , then $\sum_{j=2}^{I} \omega_{j}$ takes values in the set $\{-I+1,-I+3, \ldots, I-1\}$. A realization $\sum_{j=2}^{I} \omega_{j}=k, k \in\{-I+1,-I+3, \ldots, I-1\}$, has a probability of occurrence equal to that of $\frac{1}{2}(I+k-1)$ successes in $I-1$ Bernoulli trials, where a success is defined by an $\omega_{i}$ 's taking the value of 1 . With the probabilities being computable in terms of the binomial distribution, we may compute the probabilities for the equilibrium state of the economy when individuals' decisions are given by Equ. (25) and (26). For this, it suffices that the probability Prob $\left\{\omega_{1}\right\}$ be known as a function of fundamentals. We obtain the following by means of elementary but tedious manipulations, which are given in the Appendix:

$$
\begin{gathered}
\operatorname{Prob}\left\{\omega_{1}=1\right\} \\
=\frac{\sum_{k} \operatorname{Prob}\left\{\omega_{1}=1 \mid \sum_{i=2}^{I} \omega_{i}=k\right\} \cdot \operatorname{Prob}\left\{\sum_{i=2}^{I} \omega_{i}=k \mid \omega_{1}=-1\right\}}{1-\sum_{k} \operatorname{Prob}\left\{\omega_{1}=1 \mid \sum_{i=2}^{I} \omega_{i}=k\right\} \cdot\left[\operatorname{Prob}\left\{\sum_{i=2}^{I} \omega_{i}=k \mid \omega_{1}=1\right\}-\operatorname{Prob}\left\{\sum_{i=2}^{I} \omega_{i}=k \mid \omega_{1}=-1\right\}\right]} .
\end{gathered}
$$

It is straightforward to establish that the expression on the r.h.s. is positive and less than 1. The probabilities for all possible configurations may be computed. That is,

$$
\operatorname{Prob}\left\{\omega_{1}, \omega_{2}, \ldots, \omega_{I}\right\}=\operatorname{Prob}\left\{\omega_{1}\right\} \prod_{i=2}^{I} \operatorname{Prob}\left\{\omega_{i} \mid \omega_{1}\right\},
$$

where Prob $\left\{\omega_{1}\right\}$ is given from (27) and Prob $\left\{\omega_{i} \mid \omega_{1}\right\}$ from (26).

The respective mean states may also be computed. They would not, of course, coincide with those from the case where individuals' expected utilities depend upon the expectations of their neighbors' states. The differences in expected utilities between the former and the latter cases may be interpreted as the expected value of perfect information over neighbors' decisions. 


\subsection{An Econometric Interpretation}

The model of discrete decisions with social interactions, when utilities depend on the actual realizations of neighbors' decisions, admits an interpretation as an econometric model of simultaneous equations involving discrete decisions. ${ }^{12}$ Specifically, if the utility functions of agents $i=1, \ldots, I$, as defined in (7) and (8), are interpreted as indicator functions for the discrete choices of the logit models, then the discrete choice probabilities, given by (25) and (26), are the corresponding reduced forms for the system of simultaneous equations of the logit type.

This remark is timely. The recent resurgence of interest in estimating models of discrete games with multiple equilibria has led to econometric models similar to these. Tamer $(2002 ; 2003)$ poses models of strategic games that allow one to estimate multiple equilibria. In contrast, the earlier literature on structural models of discrete choice, such as Schmidt (1981) and others, emphasizes conditions for "internal consistency" or "coherency." Such conditions guarantee that given the values of exogenous variables, observed and unobserved, unique values for the dependent variables are implied and the associated likelihood functions are well defined.

We may think of decisions by an interacting group of $I$ agents with a more general interaction topology instead of the Walrasian star. The counterpart of (25) and (26), may be written in concise vector form as follows:

$$
\widetilde{\omega}=\mathbf{1}\left[2 h \mathbf{I}+2 \mathbf{N}^{-1} \mathbf{J} \boldsymbol{\Gamma} \tilde{\omega}+\widetilde{\varepsilon}\right],
$$

where $\mathbf{I}, \mathbf{N}^{-1}, \mathbf{J}$, the matrix of interaction coefficients and $\boldsymbol{\Gamma}$, the adjacency matrix of the interaction topology, are $I \times I$ matrices defined in subsection 2 , the $I$ column vector $\tilde{\varepsilon}$ is defined as the difference of $2 I$ independently and identically type I extreme-value distributed random variables, $\varepsilon_{i}=\epsilon_{i}(1)-\epsilon_{i}(-1)$, written as a column vector, $\widetilde{\varepsilon} \equiv \tilde{\epsilon}(1)-\tilde{\epsilon}(-1)$, and $\mathbf{1}[\mathcal{R}]$ is a $I$ vector indicator function of the $I$ vector $\mathcal{R}$, with its $i$ th element equal to 1 , if the $i$ th element of $\mathcal{R}, \mathcal{R}_{i}>0$, and is equal to -1 , otherwise. Equ. (29) represents a nonlinear spatial autoregressive model for discrete endogenous variables.

The consistency conditions proposed by Schmidt (1981) applied to (29) reduce to the condition that the model be recursive [ ibid., Condition 12.6, p. 429 ]. To see this, the case of Walrasian star interaction, which involves an adjacency matrix $\boldsymbol{\Gamma}_{W}$ of the form: $\boldsymbol{\Gamma}_{W}=\left[\begin{array}{cc}0 & \mathbf{1}_{I-1}^{\mathrm{T}} \\ \mathbf{1}_{I-1} & \mathbf{0}_{I-1}\end{array}\right]$, where $\mathbf{1}_{I-1}=(1, \ldots, 1)$, the column vector of 1 's of dimension $I-1$, and $\mathbf{0}_{I-1}$ the $(I-1) \times(I-1)$ matrix of 0 's. It follows that not all principal minors of $\mathbf{N}^{-1} \mathbf{J} \boldsymbol{\Gamma}$ are equal to 0 , and therefore, Condition 12.6 , ibid., is not satisfied. ${ }^{13}$

\subsubsection{Multiplicity of Equilibria}

As Tamer (2003), shows, if one does not insist on coherency, which was employed by earlier researchers to ensure unique outcomes, then social interactions econometric models may accommodate

\footnotetext{
${ }^{12}$ I am grateful to Chuck Manski for directing my attention to this interpretation.

${ }^{13}$ Neither is the system recursive for the cases of complete, cyclical, and path interaction topologies.
} 
economic models with multiple equilibria. The purpose of imposing coherency is to eliminate multiplicity of equilibria. That is, one wants to know whether unique or multiple social outcomes are associated with a given set of parameter values and values of stochastic shocks. Therefore, what may be desirable of some regression models, like those examined in ibid., is undesirable of models of social interactions. That is, recursiveness would be undesirable in general, because it would imply that a single agent's decisions would determine those of all others'. This may well be a feature of certain social interaction settings but should not be required of all. Furthermore, multiplicity of equilibria may be interesting in their own right. In econometric practice, one needs to determine how to handle possible multiplicities. ${ }^{14}$

Two observations are in order. First, enumeration of multiplicity of equilibria is appropriate for finite numbers of agents. Second, any expressions for the probabilities of particular outcomes, like for Prob $\left\{\omega_{1}=1\right\}$, obtained in (27) above for the case of the Walrasian star, is the equilibrium value for the marginal probability of agent 1's decisions, possibly associated with multiple outcomes. Therefore, such probabilities cannot be specified independently, in a regression setting. They are no longer in the logit form. The solution that we obtained coincides with the model's reduced form. Given appropriate data, Equ. (28) may be used to estimate the model's fundamentals be means of maximum likelihood. And third, as Soetevent (2003) underscores, the multiplicity of equilibria is entirely due to the social interactions component of individual decisions. That is, if all $J_{\mathrm{s}}$, the interaction coefficients, are equal to 0, then the number of equilibria, as enumerated by Equ. (5), in ibid., are given by $\sum_{\ell=1}^{2^{I}}\left[\Pi_{i=1}^{I} 1\left(2 h+\varepsilon_{i, \ell}>0\right)^{\frac{1+\omega i, \ell}{2}} 1\left(2 h+\varepsilon_{i, \ell} \leq 0\right)^{\frac{1-\omega i, \ell}{2}}\right]$. This expression is equal to 1 . It is straightforward to adapt this expression for the cases of the stylized topologies of social interactions examined in this paper. This is straightforward for the case of cyclical interactions, for which the expression is symmetrical. For example, the number of equilibria in that case is given by

$$
\sum_{\ell=1}^{2^{I}}\left[\Pi_{i=1}^{I} 1\left(2 h+J\left(\omega_{i-1, \ell}+\omega_{i+1, \ell}\right)+\varepsilon_{i, \ell}>0\right)^{\frac{1+\omega i, \ell}{2}} 1\left(2 h+J\left(\omega_{i-1, \ell}+\omega_{i+1, \ell}\right)+\varepsilon_{i, \ell} \leq 0\right)^{\frac{1-\omega i, \ell}{2}}\right] .
$$

The number of equilibria for this and the other stylized cases will be discussed further in a later version of this paper.

\section{Dynamic Analysis of Social Interactions}

In moving to a dynamic analysis with social interactions in nonlinear settings, we wish to emphasize the role of expectations and to distinguish between the particular impact of the topology of interactions and that of the nonlinearity of the model. We approach the dynamic case as follows. We start with the general case where each agent makes a decision with knowledge of the actual state of her neighbors in the previous period, given an arbitrary interaction topology. This is novel analysis in the context of the social interactions literature. We provide a general theorem for general social interactions topologies that establishes the existence of a stationary distribution and

\footnotetext{
${ }^{14}$ Kooreman and Soetevent (2002) assume that all possible multiple equilibria are equally likely. Tamer (2003), on the other hand, groups multiple outcomes.
} 
provide a specific result for the case of cyclical interactions. The complexity of the general case suggests that it is interesting to examine the vastly simplifying case when individuals are assumed to make decisions under the assumption that their expectations of their neighbors's decisions are to those agents' mean choices in the previous period. This approach to dynamics provides important insight into the general case. It allows us to generalize the Brock-Durlauf mean field model, which continue to serve as a fundamental building block for the analysis of the other topologies we have have examined in detail above. We see that the dynamics differ substantially between the mean field case and the other topologies that exhibit local interaction.

\subsection{Stochastic Dynamics for General Topologies}

First we deal with the general case, where the state of the economy at time $t, \tilde{\omega}_{t}$, depends upon the actual decision of each agent's neighbors in period $t-1$. This follows readily from each agent's making her decision at time $t$, given her knowledge of her neighbors' actual decisions at time $t-1$, $\widetilde{\omega}_{\nu(i), t-1}$, defined as the subvector of $\tilde{\omega}_{t-1}$ that pertains to agent $i$ 's neighbors. That is, adapting Equ. (3) in a dynamic setting, we have:

$$
\left.\operatorname{Prob}\left(\omega_{i, t}=1\right) \mid \tilde{\omega}_{\nu(i), t-1}\right)=\frac{\exp \left[\beta\left(2 h+2 \frac{1}{|\nu(i)|} \sum_{j \in \nu(i)} J_{i j} \omega_{j, t-1}\right)\right]}{1+\exp \left[\beta\left(2 h+2 \frac{1}{|\nu(i)|} \sum_{j \in \nu(i)} J_{i j} \omega_{j, t-1}\right)\right]} .
$$

The dynamic counterpart of the static model of interactions based on agents' actual environments, examined in Section 3, by adopting the concise notation of Equ. (29), may be written

$$
\tilde{\omega}_{t}=\mathbf{1}\left[2 h \mathbf{I}+2 \mathbf{N}^{-1} \mathbf{J} \boldsymbol{\Gamma} \tilde{\omega}_{t-1}+\tilde{\varepsilon}_{t}\right] .
$$

This notation underscores that the interaction topology affects the dynamics via the properties of the adjacency matrix $\boldsymbol{\Gamma}$ and of the matrices containing the number of each agent's neighbors, $\mathbf{N}^{-1}$, and the interaction coefficients, $\mathbf{J}$.

Let us consider the state of the economy in two successive periods. For each of the $2^{I}$ possible realizations of $\tilde{\omega}_{t-1}, \tilde{\omega}_{t-1} \in \underbrace{\{-1,1\} \times \ldots \times\{-1,1\}}_{I}$, Equ. (30) defines conditional choice probabilities for each agent in each of the models of social interaction, which are in effect transition probabilities for each of the $2^{I}$ possible realizations of $\tilde{\omega}_{t}$, given $\tilde{\omega}_{t-1}$. The dynamic counterparts of Equ. (5), (9)-(10) and (12) are special cases of this definition.

The state of the economy evolves according to a Markov stochastic process which is defined from the finite (but large, if $I$ is large) sample space $\underbrace{\{-1,1\} \times \ldots \times\{-1,1\}}_{I}$, into itself and has fixed transition probabilities. That is, the transition probability from $\tilde{\omega}_{t-1}=\tilde{\omega}^{\prime}$ to $\tilde{\omega}_{t}=\tilde{\omega}^{\prime \prime}$ is equal to

$$
\prod_{i=1}^{I} \operatorname{Prob}\left\{\omega_{i, t}=\tilde{\omega}_{i}^{\prime \prime} \mid \tilde{\omega}_{\nu(i), t-1}=\tilde{\omega}_{\nu(i)}^{\prime}\right\}
$$


These probabilities sum to 1 , when the sum is taken over all possible realizations of $\tilde{\omega}^{\prime \prime}$, for any given $\tilde{\omega}^{\prime}$. Put differently, the above expression is the entry for row $\tilde{\omega}^{\prime}$ and column $\tilde{\omega}^{\prime \prime}$ of the $2^{I} \times 2^{I}$ stochastic transition matrix of the Markov process.

Taking cues from by Asavathiratham (2000) [ see also Asavathiratham et al. (2001) ] allows us to transform the general case (30) to a form that is amenable to analysis by means of the standard tools for Markov processes. To see how this may be done, consider representing the state of agent $i$ instead of the binary set of outcomes $\{-1,1\}$, by the row vector $(10)$, if $\omega_{i}=1$, and by the row vector $(01)$, if $\omega_{i}=1$. In this fashion, a realization $\tilde{\omega}$ may be represented by a row vector

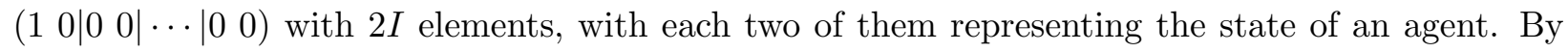
stacking up all possible realization vectors we obtain a $2^{I} \times 2 I$ matrix, known as the event matrix [ Asavathiratham (2000), p. 109 ], which represents all possible states of the economy. Equ. (30) allows us to define a $2^{I} \times 2^{I}$ transition matrix, to be denoted by $\mathcal{H}$, which is a stochastic matrix that expresses the transition probabilities for the Markov process that describes the evolution of the state of the economy from $\tilde{\omega}_{t-1}$ to $\tilde{\omega}_{t}$. Next, we define the column vector $\Psi_{t}$, with $2^{I}$ rows, and entries all zeroes, except one, which is 1 and indicates that the economy is at the corresponding state, that is associated with the respective row of the event matrix. Accordingly, we may define a probability distribution over the possible states of the economy, the rows of the event matrix. Clearly, a stationary probability distribution over the states of the economy, if it exists, is given by a positive eigenvector of $\mathcal{H}, \tilde{\Psi}$, suitably normalized, that satisfies

$$
\mathcal{H} \tilde{\Psi}=\tilde{\Psi}
$$

Since $\mathcal{H}$ is a stochastic matrix, its dominant eigenvalue (the Perron-Frobenius root) is 1 , and such a vector always exists. As Asavathiratham discusses [op. cit., Theorem 5.7, p. 109], the stationary distribution might not be unique, if the corresponding dominant eigenvalue that is equal to 1 does not have an algebraic multiplicity of 1 . Because of the nature of the interaction topology, some of the states of the economy may not communicate, and the process might not have a unique recurrent class.

There is another payoff from working with the stationary distribution associated with an economy where agents act with knowledge of their neighbors' actual decisions. It coincides with the equilibrium distribution in the static case when interactions are based on agents' actual environments, discussed in section 3 above. We rely on the intuitive appeal of this claim, which follows from the definition of the agents' optimal decisions and the associated probability distributions that they imply, that is Equ. (20). ${ }^{15}$ However, this particular description of the equilibrium distribution is rather unwieldy and it is for this reason that we turn to special cases that are amenable to exact solution.

\footnotetext{
${ }^{15}$ For a proof, one may adapt the classic treatment of Preston (1974), p. $10-18$.
} 


\subsubsection{Cyclical Interaction Revisited}

When the interaction structure is translation-invariant, that is when only relative distance between agents matter and not their actual locations, and if the process is reversible, that is, the interaction structure is symmetric so that the process may be reversed, just like a movie being played backwards and still making sense, then an important result follows. Drawing from a recent paper by Bigelis et al. (1999) we state (without proof) their result for the existence of a stationary distribution and the closed form it actually assumes, in the proposition that follows. Here, we write the interaction effect from $j$ to $i$ as $J(i-j)$ so as to conform to the statement of the results in ibid., p. 3936.

Proposition 6. Let the total number of agents $I$ be finite and $\Lambda$ be a d-dimensional torus containing $L^{d}$ lattice sites (that is, $\Lambda$ is a cube in $Z^{d}$ containing $L^{d}$ points and having periodic boundaries). If the Markov process for $\tilde{\omega}_{t}$, defined on the configuration space $\{-1,1\}^{\Lambda}$, with fixed transition probabilities, given by:

$$
\operatorname{Prob}\left\{\omega_{i, t}=\varpi \mid \tilde{\omega}_{t-1}\right\}=\frac{1}{2}\left[1+\varpi \tanh \left(\beta \sum_{j \in \Lambda} J(i-j) \omega_{j, t-1}+\beta h\right)\right], \varpi \in\{-1,1\},
$$

is reversible, for which it suffices that $J(i-j)=J(-i+j)$, then the process (32) has a unique stationary distribution given by:

$$
\operatorname{Prob}\{\tilde{\omega}\}=\bar{\Pi}^{-1} \prod_{i \in \Lambda} e^{\beta h \omega_{i}} \cosh \left(\beta \sum_{j \in \Lambda} J(i-j) \omega_{j}+\beta h\right),
$$

where $\bar{\Pi}$ is a normalizing constant.

It is clear from the above expression for the stationary distribution that when individuals are conformist, neighbors' making similar decisions strengthen the likelihood that an agent would conform. The cases of global interactions and circular interactions with symmetric interaction coefficients, $J_{i-1}=J_{i+1}$, are translation-invariant, and under the assumption of reversibility, they become special cases of the above theory. This result encompasses our finding in section 3.1 as a special case.

\subsubsection{The Walrasian Star Revisited}

The Walrasian star model, although not translation-invariant, is, however, amenable to specific treatment, based first principles. Following up on subsection 3.2 above, when agents are influenced by the actual decisions of their neighbors, the evolution of the economy may be defined as:

$$
\begin{gathered}
\operatorname{Prob}\left\{\omega_{1, t}=1 \mid \sum_{j=2}^{I} \omega_{j, t-1}=\Omega_{-1, t-1}\right\}=\frac{\exp \left[\beta h+\frac{1}{I-1} \beta J_{S} \Omega_{-1, t-1}\right]}{1+\exp \left[\beta h+\frac{1}{I-1} \beta J_{S} \Omega_{-1, t-1}\right]}, \\
\operatorname{Prob}\left\{\Omega_{-1, t}=K \mid \omega_{1, t-1}=\omega_{1}\right\}=B\left(\frac{1}{2}(I+K-1), I-1 ; \operatorname{Prob}\left\{\omega_{i, t}=1 \mid \omega_{1, t-1}\right\}\right), \\
K \in\{-I+1,-I+3, \ldots, I-1\},
\end{gathered}
$$


where $\Omega_{-1, t}=\sum_{j=2}^{I} \omega_{j, t}$ and $B\left(\frac{1}{2}(I+K-1), I-1 ; \operatorname{Prob}\left\{\omega_{i, t}=1 \mid \omega_{1, t-1}\right\}\right)$ denotes the value of the binomial distribution for $\frac{1}{2}(I+K-1)$ successes in $I-1$ Bernoulli trials, with each success having probability given by

$$
\operatorname{Prob}\left\{\omega_{i, t}=1 \mid \omega_{1, t-1}\right\}=\frac{\exp \left[\beta h+\beta J \omega_{1, t-1}\right]}{1+\exp \left[\beta h+\beta J \omega_{1, t-1}\right]} ; i=2, \ldots, I .
$$

Equ. (34) and (36) are the transition probability of a Markov process in terms of the pair of discretevalues random variables, $\left(\omega_{1, t}, \Omega_{-1, t}\right)$, where defined over the sample space $\{-1,1\} \times\{-I+1,-I+$ $3, \ldots, I-1\}$. It is straightforward to show that the stationary probability for the state of agent 1, Prob $\left\{\omega_{1}=1\right\}$ distribution coincides with that given by Equ. (27) above and therefore for the state of the economy by Equ. (28). This is not a coincidence, as we discussed earlier.

Obviously, the general treatment discussed earlier is quite unwieldy, except for some special cases, because it is very difficult to obtain the spectral properties of the above transition matrix directly. We have been able to solve for the equilibrium distributions in the cases of cyclical interaction and for the Walrasian star. Unfortunately, it is impossible to make additional progress in understanding qualitative properties of the dynamics, even if we are very specific about the interaction topology, by means of this very general description. It is for this reason that it pays to study dynamics under the assumption that each individual's expectation of her neighbors' choice in the current period is equal to those agents' mean choice in the previous period.

We see next how our approach adapts the Brock-Durlauf mean field model to richer interaction structures and continues to lead to multiple equilibria. As Blume and Durlauf (2002) show for the Brock-Durlauf model and for other models, these multiple equilibria characterize local extrema of the stationary probability distribution functions, with the stable asymmetric equilibria being local maxima and the symmetric unstable equilibrium being a local minimum.

\subsection{Dynamics with Expectations Based on Lagged Mean Decisions of Neighbors}

Here we work with the assumption that each individual's expectation of her neighbors' choice at time $t$ is equal to those agents' mean choice at time $t-1, \mathcal{E}_{i}\left\{\omega_{j, t}\right\}=m_{j, t-1}$. This allows us to examine dynamics for the stylized topologies that we have been working with as well as to obtain results for the case of general interaction topologies by working with nonlinear deterministic difference equations.

We use again as a benchmark the mean field case of Brock and Durlauf, op. cit. for which Equ. (5) allows us to write a nonlinear deterministic difference equation for the evolution of expected choice by the typical agent $m_{t}$, for the complete interactions model:

$$
m_{t}=\tanh \left(\beta h+\beta J m_{t-1}\right) .
$$

Working in like manner with (9)-(10) and (12) we obtain for the Walrasian-star model:

$$
m_{1, t}=\tanh \left(\beta h+\beta J_{S} m_{-1, t-1}\right) ;
$$




$$
m_{-1, t}=\tanh \left(\beta h+\beta J m_{1, t-1}\right) ;
$$

for the cyclical interaction model:

$$
m_{i, t}=\tanh \left(\beta h+\frac{1}{2} \beta\left(J_{B} m_{i-1, t-1}+J_{F} m_{i+1, t-1}\right)\right), i=1, \ldots, I
$$

and for the path interaction model:

$$
\begin{gathered}
m_{-L, t}=\tanh \left(\beta h+\beta J_{F} m_{-L+1, t-1}\right) \\
m_{i, t}=\tanh \left(\beta h+\frac{1}{2} \beta\left(J_{B} m_{i-1, t-1}+J_{F} m_{i+1, t-1}\right)\right),-L+1 \leq i \leq L-1 . \\
m_{L, t}=\tanh \left(\beta h+\beta J_{B} m_{L-1, t-1}\right) .
\end{gathered}
$$

As we discuss above, with symmetry, $J=J_{S}=J_{F}=J_{B}$, then the above equations imply the same steady state equation, which coincides with that of the Brock-Durlauf mean field theory case, Equ. (5). To understand how the interaction topology affects the dynamics of adjustment to a steady state even when we do impose symmetry, we need to perturb the steady state equilibrium. We set ideas with a brief analysis of the local dynamics around the steady states of the BrockDurlauf mean field model and then move to other topologies.

\subsubsection{Dynamics of The Brock-Durlauf Mean Field Model}

We recall that complete pairwise interactions are equivalent to the We briefly reproduce here the Brock-Durlauf analysis of the mean field case, which serves as a benchmark. See Brock and Durlauf, op. cit., p. 12-14, for more details. Consider Equ. (37), and use $m^{*}$ to denote a steady state. Note that the steady state equilibrium condition coincides with the equilibrium of the static model. Let $\Delta m_{t} \equiv m_{t}-m^{*}$ denote the deviation near the steady state. Linearization of Equ. (37) yields:

$$
\Delta m_{t}=\beta J \tanh ^{\prime}\left(\beta h+\beta J m^{*}\right) \Delta m_{t-1} .
$$

Therefore, local stability depends entirely upon the magnitude and sign of the coefficient of $\Delta m(t-$ 1) above, that is $\beta J \tanh ^{\prime}\left(\beta h+\beta J m^{*}\right)$.

The results may be summarized as follows. Considering for concreteness the case of $\beta J>1$, and setting for simplicity $h=0$, in which case the symmetric steady state is $m^{*}=0$, and in addition, for the asymmetric ones, the upper is positive and the lower is negative, $m_{+}^{*}, m_{-}^{*}$. The steady states exhibit "symmetry breaking": the symmetric is unstable, $\beta J \tanh ^{\prime}(0)>1$, while the asymmetric ones are stable:

$$
\begin{aligned}
& \beta J \tanh ^{\prime}\left(\beta h+\beta J m_{+}^{*}\right)<1 ; \\
& \beta J \tanh ^{\prime}\left(\beta h+\beta J m_{-}^{*}\right)<1 .
\end{aligned}
$$

It should be noted that although individuals may be in either of the two realizations of the discrete state, the dynamic adjustment was defined earlier in terms of the expected choice of neighbors in the previous period. In that case, when self-consistent expectations exist in the 
Brock-Durlauf model, then Proposition 6, ibid., guarantees that the sample average population choice converges weakly to the self-consistent expectation, the steady state equilibrium solutions of (37). Blume and Durlauf (2002) examine the stochastic dynamics of this model. They show that when individuals revise their choices at independent random times given by a "Poisson" clock by looking at all other agents' lagged choices and if their number is large, then the mean decision obeys the continuous-time counterpart of the mean-field equation Equ. (37).

\subsubsection{Dynamics of the Walrasian-Star}

Working next for the Walrasian-star case, linearization of Equ. (38)-(39) around a steady state $\left(m_{1}^{*}, m_{-1}^{*}\right)$ yields a two-dimensional system:

$$
\left[\begin{array}{c}
\Delta m_{1, t} \\
\Delta m_{-1, t}
\end{array}\right]=\left[\begin{array}{cc}
0 & \beta J_{S} \tau_{1} \\
\beta J \tau_{-1} & 0
\end{array}\right]\left[\begin{array}{c}
\Delta m_{1, t-1} \\
\Delta m_{-1, t-1}
\end{array}\right],
$$

where $\tau_{1} \equiv \tanh ^{\prime}\left(\beta h+\beta J m_{-1}^{*}\right)$ and $\tau_{-1} \equiv \tanh ^{\prime}\left(\beta h+\beta J m_{1}^{*}\right)$.

Working in the usual fashion, we obtain the characteristic equation for Equ. (45), whose roots are the eigenvalues of the matrix in the R.H.S. of (45), which satisfy $\lambda^{2}=\tau_{1} \tau_{-1} \beta^{2} J J_{S}$. Therefore, if $J J_{S}>0$, and since $\tau_{1}, \tau_{-1}>(<) 0$, as $\beta>(<) 0$, then the eigenvalues are real and have equal magnitudes but opposite signs: $|\lambda|=\beta \sqrt{\tau_{1} \tau_{-1} J J_{S}}$. If, on the other hand, $J J_{S}<0$, then the eigenvalues are conjugate imaginary. In either case, we have that if the economy is near an asymmetric equilibrium for the agents outside the center and for the agent in the center, $\beta \tau_{1}\left|J_{S}\right|<1$, and $\beta \tau_{-1}|J|<1$, the eigenvalues have absolute values less than 1 , and the dynamic adjustment is stable. If, on the other hand, the economy is near the symmetric equilibrium for all agents, $\beta \tau_{1}\left|J_{S}\right|>1$, and $\beta \tau_{-1}|J|>1$, the eigenvalues have absolute values greater than 1 , and the dynamic adjustment is unstable. Finally, if the economy is near an asymmetric equilibrium for the agents outside the center and the symmetric one for the agent in the center, $\beta \tau_{1}\left|J_{S}\right|<1$, and $\beta \tau_{-1}|J|>1$, the eigenvalues may have absolute values greater than 1 , depending upon parameter values, and the dynamic adjustment would be unstable. The presence of factor $\beta \sqrt{\tau_{1} \tau_{-1}}$ carries the impact of the nonlinearity of the dynamics for the approximate model. Again, the instability of the symmetric steady state is more pronounced the larger are $\beta \tau_{1}$ and $\beta \tau_{-1} \cdot{ }^{16}$

\subsubsection{Dynamics of Cyclical Interaction}

Working next for the cyclical interaction case, linearization of Equ. (40) around its isotropic steady state $m^{*}$, that is, one of the solutions of (13), yields:

$$
\Delta m_{i, t}=\tanh ^{\prime}\left(\beta h+\frac{1}{2} \beta\left(J_{B} m_{i-1}^{*}+J_{F} m_{i+1}^{*}\right)\right)
$$

\footnotetext{
${ }^{16}$ We note that the dynamics of the model continue to reflect properties of both the topology of interaction and the nonlinearity. This is somewhat obscured by the fact that the product of the eigenvalues of the adjacency matrix, $-(I-1)$, cancels out because of the division by $I-1$.
} 


$$
\times\left[\frac{1}{2} \beta J_{B} \Delta m_{i-1, t-1}+\frac{1}{2} \beta J_{F} \Delta m_{i+1, t-1}\right], i=1, \ldots, I .
$$

Let $\Delta \mathbf{m}_{t}:=\left(\Delta m_{1, t}, \ldots, \Delta m_{I, t}\right)^{\mathrm{T}}$; This above system, for the case of $J_{F}=J_{B}=J$, may be written, equivalently as:

$$
\Delta \mathbf{m}_{t}=\beta \tau^{C} J \cdot \frac{1}{2} \boldsymbol{\Gamma}_{C} \cdot \Delta \mathbf{m}_{t-1},
$$

where $\tau^{C}:=\tanh ^{\prime}\left(\beta h+\frac{1}{2} \beta\left(J m_{i-1}^{*}+J m_{i+1}^{*}\right)\right)$. The adjacency matrix $\boldsymbol{\Gamma}_{C}$ has as rows the permutations of $(0,1,0, \ldots, 1)$. The general solution to equation (47) can be written, in the usual fashion for linear systems and after a number of tedious steps [ see Appendix, section 8.8 ], as a linear combination of the eigenvectors, each multiplied by its respective eigenvalue raised to the power of $t$, and weighted by arbitrary constants, which are determined by initial conditions. Specifically, let us assume that $I$ is odd and therefore write:

$$
\begin{gathered}
{\left[\begin{array}{c}
\Delta m_{1, t} \\
\cdot \\
\cdot \\
\cdot m_{I, t}
\end{array}\right]=A_{1}\left(\beta J \tau^{C}\right)^{t}\left[\begin{array}{c}
1 \\
\cdot \\
\cdot \\
\cdot \\
1
\end{array}\right]+A_{2}\left(\beta J \tau^{C} \cos \left(\frac{2 \pi}{I}\right)\right)^{t}\left[\begin{array}{c}
\cos \left(\frac{2 \pi}{I}\right) \\
\cos \left(\frac{4 \pi}{I}\right) \\
\cdot \\
\cdot \\
1
\end{array}\right]} \\
+A_{3}\left(\beta J^{C} \cos \left(\frac{2 \pi}{I}\right)\right)^{t}\left[\begin{array}{c}
\sin \left(\frac{2 \pi}{I}\right) \\
\sin \left(\frac{4 \pi}{I}\right) \\
\cdot \\
\cdot \\
0
\end{array}\right]+\ldots,
\end{gathered}
$$

where $A_{1}, \ldots$, denote constants which are computed from initial conditions.

To see the implications of this model, consider that the system is originally at a steady state equilibrium when it is shocked at time 0 by changing agent 1's decision, say $\Delta m_{1,0}=1, \Delta m_{2,0}=$ $\ldots, \Delta m_{I, 0}=0$. We thus have $I$ equations (48) in the unknown constants $A_{1}, \ldots, A_{I}$, that reflect the initial conditions. These equations may be solved uniquely since the $I$ eigenvectors span the space.

The solution (48) implies oscillatory behavior in a spatial sense. If the factor $\beta J \tau^{C}$ were not present, as in the linear case, as $t$ tends to $\infty$, the system would tend to the eigenvector corresponding to the maximal eigenvalue, which in that case would be 1 . The corresponding eigenvector is $(1,1, \ldots, 1)^{\mathrm{T}}$. The economy would exhibit persistence, in that case. However, the factor $\beta J \tau^{C}$ changes this. The amplitude of the oscillations has a maximum given by the maximal eigenvalue $\beta J \tau^{C}$. The oscillations range from $\beta J \tau^{C}$ to $-\beta J \tau^{C}$, depending upon the position of an agent on the circle. If, as we assumed, the economy starts from an isotropic equilibrium, then the eventual pattern of states would be determined by whether the starting isotropic equilibrium is a stable or unstable one. If all agents start from a stable equilibrium, then these amplitudes all have absolute values less than 1 . It follows that the amplitudes of the oscillations diminish over time, 
and the economy tends to return to the same isotropic steady state that it started from. If, on the other hand, the economy starts from the unstable isotropic equilibrium, $\beta J \tau^{C}>1$. Because the magnitude of the oscillations varies spatially, depending upon the position of an agent on the circle, and is bounded upwards by $\beta J \tau^{C}$, even when the economy starts from the unstable equilibrium, some agents would not be changing their decisions.

The system (46) can be studied further, even if $J_{F} \neq J_{B}$, because the matrix is circulant and its diagonalization is accomplished by means of the Fourier matrix that diagonalizes all circulant matrices [ Davis (1979), p. 73; Brockwell and Davis (1991), p. 135 ] The solution in the general case of $J_{F} \neq J_{B}$, involves, intuitively, two "trains" of $I$ oscillatory terms with different amplitudes, indexed by the individuals and modulated by time-varying amplitudes, which move in opposite directions around the circle. In either case, that is, in either the general or the specific case, the spatial fluctuations can be interpreted as cluster emergence.

How will the economy move if it starts with some of the agents in either of the asymmetric equilibria and others at the symmetric one? Roughly speaking, the evolution of the components of the economy when adjacent sets of agents are perturbed away from the same isotropic equilibrium may be handled by means of the above techniques, applied to the model of path interaction, which we discuss further below. The properties of the global dynamics are much harder to characterize.

Cyclical interactions are ubiquitous in social settings but imply no obvious empirical tests. Support for the model's predictions comes from computational results obtained by Fujita, Krugman and Venables (1999) and Epstein and Axtell (1996), although those models have different motivations and structures. In the latter, the sugarscape economy has also been adapted to a ring world, by which it is meant that agents interact in a cyclical fashion. The Epstein-Axtell simulations show, ibid., p. 170-176, that agents who can move tend to cluster into groups, often of comparable size. They interpret this outcome as seemingly social behavior, which "is not driven by any social impulse but is solely a product of the agent-environment coupling" [ ibid., p. 172 ].

Our results are also consistent with Danny Quah's findings on cluster emergence in continuous spatial settings, which occurs for reasons that are identical to ours [ Quah (1999; 2000) ]. Technically, the similarity of these results originates in the fact that for consistency, the solution must obey cyclical symmetry, which brings us to circulant matrices, whose eigenvalues involve the complex roots of 1. In contrast, Quah works with Toeplitz operators, which are the continuous time and space counterparts of circulant matrices. Aside from such obvious differences from Quah's model as his use of continuous space and time, the present model has a different implication that is entirely due to the multiplicity of steady state equilibria. Once disturbed, all agents in the system will ultimately return to a steady state, which may be either the upper or the lower, as the symmetric one is unstable. This feature is particularly important in our case and distinguishes our results from those of Quah's. While after the symmetric steady state equilibrium is disturbed, adjustment to a new steady state is associated, as in Quah's case, with spatial clustering, where some individuals may end up in the positive and other in the negative steady states. However, unlike Quah's case, clustering here is permanent. This possibility is entirely due to the multiplicity of steady 
state equilibria in our model, unlike uniqueness of equilibrium in Quah's model. We note that our results are broadly consistent with those by Elkhader (1992), who studies the general properties of bounded orbits of deterministic systems of differential equations roughly corresponding to our cyclical interaction case. Elkhader shows that the omega limit set of such orbits contains a steady state or a nonconstant periodic orbit.

\subsubsection{Small versus Large Neighborhoods}

Does the speed of adjustment vary with neighborhood size? We can address this question by extending the range of local interactions in the model that leads to Equ. (40) so that agent $i$ is influenced by agents $\left\{i-L_{n}, \ldots, i-1, i+1, \ldots, i+L_{n}\right\}, L_{n} \leq I-1$. We set $J_{B}=J_{F}$. The counterpart of Equ. (40) implies that the cyclical interaction model with larger neighborhoods possesses the same steady states as the solutions of (13). The counterpart of Equ. (46) becomes:

$$
\begin{gathered}
\Delta m_{i, t}=\tanh ^{\prime}\left(\beta h+\frac{1}{2 L_{n}} \beta J\left(\sum_{\ell=1}^{L_{n}}\left[m_{i-\ell}^{*}+m_{i+\ell}^{*}\right]\right)\right) \\
\times \beta J \frac{1}{2 L_{n}}\left[\sum_{\ell=1}^{L_{n}}\left[\Delta m_{i-\ell, t-1}+\Delta m_{i+\ell, t-1}\right]\right], i=1, \ldots, I .
\end{gathered}
$$

The counterpart of Equ. (47) for Equ. (49) involves a real symmetric circulant matrix $\boldsymbol{\Gamma}_{C N}$, whose eigenvalues and eigenvectors are known in closed form [ Proposition 4.5.1, p. 134-135, Brockwell and Davis (1991) ]. ${ }^{17}$ Let us define $\tau_{S} \equiv\left(\beta h+\frac{1}{2 L} \beta J\left(\sum_{\ell=1}^{L}\left[m_{i-\ell}^{*}+m_{i+\ell}^{*}\right]\right)\right) \beta J$. The eigenvalues and eigenvectors of matrix $\frac{1}{2} \boldsymbol{\Gamma}_{C N}$ again involve sine and cosine terms. The maximal eigenvalue is equal to 1 , and the corresponding eigenvector is $(1,1, \ldots, 1)$. The remaining eigenvalues come in pairs, if $I$ is odd, or there are $\frac{I-2}{2}$ pairs of double eigenvalues and an additional distinct one, if $I$ is even.

The speed of adjustment associated with this model may be compared analytically between two benchmark cases, namely the case of complete (global) interaction $\left(L_{n}=I-1\right)$, and the case of the smallest possible neighborhood, which in this case is given by the cyclical interaction model examined earlier. The local dynamics of both those models near steady states possess the same maximal eigenvalue. ${ }^{18}$ Therefore, the speed of adjustment to the steady state is determined by the second largest eigenvalue. This is equal to $\beta J \tau^{C} \cos \left(\frac{2 \pi}{I}\right)$, for the cyclical interaction, and to $\frac{-1}{I-1} \beta J \tau^{C}$, for the complete interaction topologies, respectively. The former is absolutely larger

\footnotetext{
${ }^{17}$ From Davis (1979), p. 72-73, we have that all circulant matrices of the same order have the same set of (right) eigenvectors, the columns of $F^{*}$, ibid., 32. Let a circulant matrix be defined by rows being permutations of $\left(c_{1}, c_{2}, \ldots, c_{I}\right)$. Its eigenvalues are given by $\lambda_{j}=\phi\left(\frac{2 \pi}{I}(j-1)\right), j=1,2, \ldots, I$, where

$$
\phi\left(\frac{2 \pi}{I}(j-1)\right) \equiv c_{1}+c_{2} \exp \left[\sqrt{-1} \frac{2 \pi}{I}(j-1)\right]+\ldots+c_{I} \exp \left[\sqrt{-1} \frac{2 \pi}{I}(j-1)(I-1)\right]
$$

However, this general treatment is in practice a bit unwieldy, because it involves complex numbers, while we would to work with real eigenvalues and eigenvectors.

${ }^{18}$ This is equal to $\beta J \tau^{C}$, for the cyclical interaction, and $\frac{1}{I-1} \beta J \tanh ^{\prime}\left(\beta h+\beta J m^{*}\right)(I-1)$, for the complete interaction topologies, respectively. But, $\tau^{C}=\tanh ^{\prime}\left(\beta h+\beta J m^{*}\right)$.
} 
than the latter, for large $I$, and therefore adjustment is faster in the case of global than of local interaction, which does conform with intuition on the role of greater interconnectedness. It is an open question whether the result of Young (1998) on the role of close-knit social groups in the diffusion of innovations may be also shown to apply here.

Dynamics for more general cyclical interaction cases may be studied even when the backward and forward interaction coefficients differ, as long as the pattern of dependence gives rise to a circulant matrix. The presence of both local and global interactions, that is where agent $i$ is influenced by agents $\{i-1, i+1\}$ and by the mean state of all agents, can also be handled. It may be put in the above form and its dynamic analysis involves a circulant matrix.

\subsubsection{Dynamics of Path Interaction}

Next we consider the case of path interaction, where the agents are placed on a lattice on the real line. It is interesting that the equilibrium is still characterized by "spatial waves," as in the cyclical interaction case.

The key issue at this point is whether equilibrium is still characterized by "spatial waves," as in the circular interaction case. In fact, the question of whether this particular characteristic of social interaction is due to closure of the social structure or to the impact of local interactions may be resolved only by means of a comparison with the linear interaction case.

In working with the linear interaction case, Equ. (41) - Equ. (43), again, we linearize around an isotropic equilibrium, and write the solution [ see Appendix, section 8.9, for the technical details ]for the deviations from an isotropic steady state under symmetry:

$$
\begin{aligned}
& {\left[\begin{array}{c}
\Delta m_{-L}(t) \\
\cdot \\
\Delta m_{0}(t) \\
\cdot \\
\Delta m_{L}(t)
\end{array}\right]=A_{-L}\left(\frac{1}{2} \beta J \tau^{L} \cos \left(\frac{\pi}{2(L+1)}\right)\right)^{t}\left[\begin{array}{c}
\sin \left(\frac{\pi}{2(L+1)}\right) \\
\sin \left(\frac{\pi 2}{2(L+1)}\right) \\
\cdot \\
\cdot \\
\sin \left(\frac{\pi(2 L+1)}{2(L+1)}\right)
\end{array}\right]} \\
& +A_{-L-1}\left(\frac{1}{2} \beta J \tau^{L} \cos \left(\frac{\pi 2}{2(L+1)}\right)\right)^{t}\left[\begin{array}{c}
\sin \left(\frac{\pi 2}{2(L+1)}\right) \\
\sin \left(\frac{\pi 4}{2(L+1)}\right) \\
\cdot \\
\cdot \\
\sin \left(\frac{\pi 2(2 L+1)}{2(L+1)}\right)
\end{array}\right]+\ldots,
\end{aligned}
$$

where $, A_{-L}, \ldots, A_{L}$, denote constants which are computed from initial conditions.

There is an important, though subtle, difference from the circular interaction case, in that 1 would not be an eigenvalue of the dynamic system that describes adjustment near a steady state for interactions along a line even if the factor $\beta J \tau^{L}$ were not present. The dynamics are characterized by spatial oscillations that are again transitory but there is no relative persistence. However, the importance of this fact vanishes asymptotically, as $L \rightarrow \infty$. In fact, the case of interactions along 
an infinite line is particularly interesting and fortunately, lends itself to explicit treatment. Before turning to that immediately below, we underscore that while local interaction is responsible for clustering, closure is responsible for persistence, which we would expect with 1 being an eigenvalue in the case of circular interaction.

It is for this reason that we limit our attention to the infinite path case: $L \rightarrow \infty$. The details of the analytics are given in the Appendix, section 8.10. When we let the number of agents tend to infinity, the role of the end agents vanishes asymptotically. When we linearize around a stable isotropic steady state, the term $\left(\frac{1}{2} \beta J \tau^{L}\right)^{t}$ tends to 0 as $t$ tends to $\infty$. As the "disturbance" propagates through social interactions over time, it has a transient effect on each agent: the change originally increases, reaches a peak and then decreases. This response is like two blips that move in opposite directions away from agent 0 . However, the symmetry and the setting and intuition from the spectral theory of random fields suggest that this infinite path case should be equivalent to the infinite circle case.

In concluding the analysis of local interaction, as represented by cyclical interaction and by path interaction, we wish to underscore, once again, important similarities and differences. Dynamics in both cases involves spatial oscillations. The cyclical interaction case involves exhibits relative persistence. We see further below that relative persistence follows from regularity of social interaction.

\subsubsection{Dynamics with General Topologies}

While the qualitative differences between the various cases we examined above justify the separate treatment, we can in fact obtain a general result regarding stability, provided that we restrict the social interactions topology to be represented by a regular graph and uniform interaction structure. This is when all agents have the same number of neighbors, the same degree, $d=\nu(i)$, and the same interaction coefficients. These particular assumptions may be relaxed at the cost of notational complexity.

Working with a dynamic adaptation of (3), with each individual's expectation of her neighbors' choice at time $t$ being equal to those agents' mean choice at time $t-1$, we have:

$$
m_{i, t}=\tanh \left[\beta h+\beta \frac{1}{d} \mathbf{J} \boldsymbol{\Gamma}_{i} \mathbf{m}_{t-1}\right] i=1, \ldots, I,
$$

where $\boldsymbol{\Gamma}_{i}$ denotes the $i$ th row of the adjacency matrix, and $\mathbf{m}_{t}$ the $I$-vector consisting of the $m_{i, t}$ 's.

The stability of the system is characterized by the following proposition, which utilizes standard results based on the theory of dynamical systems and the Perron-Frobenius Theorem for symmetric positive matrices and holds for any social interactions topology.

Proposition 7.

Part I. If the topology of interaction is represented by a regular graph, whose degree is $|\nu(i)|=d$ and otherwise arbitrary, and a constant interaction coefficient $J$, then the following hold.

(a) The economy's steady states satisfy Equ. (5) and are isotropic. They are generically hyperbolic fixed points of the I-order dynamical system defined by Equ. (51). 
(b) small perturbations around a steady state $\mathbf{m}$ satisfy:

$$
\Delta \mathbf{m}_{t}=\beta \tau \frac{1}{d} J \boldsymbol{\Gamma} \Delta \mathbf{m}_{t-1},
$$

where $\tau:=\tanh ^{\prime}\left[\beta h+\beta \frac{1}{d} J \boldsymbol{\Gamma}_{i} \mathbf{m}\right]$.

(c) A necessary and sufficient condition for the local dynamics of (52) at a steady state $m_{i}=m$ to be stable (unstable) is:

$$
\beta J \tanh ^{\prime}(\beta h+\beta J m)<(>) 1 .
$$

Part II. An economy with an arbitrary topology of social interactions possesses at least one steady state.

Part III. A necessary and sufficient condition for the local dynamics of (52) at a steady state $m_{i}=m$ to be stable (unstable) is (53), even for an economy with an arbitrary topology of social interactions.

A number of remarks are in order. First, by Theorem 3.33, p. 104, Cvetković et al., op. cit., the eigenvector associated with the maximal eigenvalue is $(1,1, \ldots, 1)^{\mathrm{T}}$. As a result, the role played by this eigenvector in the qualitative discussion of circular interaction pertains to all social interactions settings represented by regular graphs, that is where all agents have the same number of neighbors. In other words, the relative persistence result that we associated above with the circular interaction topology actually applies to all regular interaction topologies. Second, isotropic steady states for the entire economy would be either stable, as when $m=m_{-}^{*}, m_{+}^{*}$, or unstable, $m=m^{*}$. Third, the nature of the time map suggests that the dynamical system (51) possesses no periodic orbits. Fourth, and most noteworthy, the analysis following Equ. (51) above may be extended, in particular, to the case where the interaction topology results from a random graph, where any two agents may be connected with probability $p$ [ Erdös and Renyi (1960) ], provided that we normalize by means of the largest possible degree, that is the size of the interaction graph, I. From Cvetković et al. (1988), p. 79, it follows that the largest eigenvalue of the adjacency matrix grows according to $p I$, as $I$ tends to infinity. The above existence and stability results carry over.

\section{Extensions}

Several possible extensions come to mind. First, it is possible to study the evolution of the second moments of individuals' decisions. But more generally, it would be interesting to analyze fully the general framework for dynamics, proposed in subsection 4.1 above, and link with the results obtained for the dynamic evolution of the mean for all topologies of interest. Second, the model pretty much as it stands admits a neural networks interpretation. Third, it would be interesting to examine the impact of the spatial extent of interactions upon the speed of adjustment. With respect to the latter, Ellison (1993) and Young (1998) have emphasized the importance of local interaction for the speed of adoption of norms and development of institutions more generally. The issue is a subtle one, in that when individuals interact mainly with small groups of neighbors, then 
"shifts of regime can occur exponentially faster than in the case of uniform interaction. [...] All else being equal, the smaller the size of the neighborhood groups, and the more close-knit they are, the faster the transition time for the whole population " [Young, op. cit., 98-99].

There are several additional ways in which the model could be extended. Two are noteworthy. First, the model may be given a production function interpretation. That is, agents' decisions may be interpreted as production decisions, and the sum of all decisions is the total output of an organization. Interactions represent synergies. Padgett (1997) also works with a production function interpretation of a cyclical interaction setting, the "hypercycle". A firm will want to design the topology of interactions in order to satisfy a profit objective, taking the synergies into consideration. This is, of course, a departure from the current setting, where we envision decentralized noncooperative behavior.

Second, additional work can model trade among agents (nations), where the interactions may be motivated as originating in pecuniary externalities associated with trade in differentiated products. Puga and Venables (1997), who explore the impact upon welfare from the creation of free trade areas, which would correspond to our complete pairwise interactions case, and "hub-and-spoke" arrangements, whereby a country liberalizes bilateral trade with several other countries, with barriers remaining among those other countries, show that the topology of pattern of trade arrangements (interactions) does matter. In view of such potential applications, it would be interesting to see the impact of interactions patterns on the persistence of center vs. periphery type phenomena among countries engaging in liberalizing trade and the sequence with which they actually entered into such arrangements.

Third, it would be interesting to further explore econometric properties of models describing systems of interacting agents, when the interaction topology is not known in advance and agents to choose whom to interact with. It would also be interesting to link with the work on endogenous network formation, as by Bala and Goyal (2000) and others.

\section{Summary and Concluding Remarks}

We examine in this paper the role of topological features of an economy's social interaction structure. Our starting point is that individuals care about the decisions of their neighbors, where the specific notion of neighborhood defines social structure. We examine the importance of specific topologies of interactions by working with fully optimized nonlinear discrete-response rules and extending the Brock-Durlauf model interactive discrete choice model to a number of stylized local interactions topologies. We examine equilibria with social interactions in both static and dynamic settings and also interpret them as models of aggregate uncertainty. When all interaction coefficients are equal, all of our models, in static settings, are qualitatively similar to the mean field case examined by Brock and Durlauf. We explore properties of a richer class of anisotropic equilibria, which appear for the case of cyclical interaction and path interaction when interaction coefficients differ.

We also examine the model where individuals make decisions on the basis of observations on 
the actual decisions of their neighbors. We obtained the equilibrium probability distributions for the case of Walrasian-star interactions, by working from first principles, and of circular interaction, by drawing on the statistical mechanics literature for the one-dimensional Ising model. We also examine the properties of the equilibrium probability distributions for the states of all agents by linking interactive discrete choice models with the econometric theory of simultaneous systems of equations modelling discrete decisions.

With local interaction either in the form of cyclical or of path interaction, synergistic effects lead to spatial oscillations and clustering, with the "most unstable" states pushing the economy away from the symmetric isotropic outcome, which is unstable. Those two interaction patterns give broadly similar results but with one important exception, namely that the cyclical interaction case is associated with greater persistence relative to the case of path interaction. We show that such greater persistence is also present in models with arbitrary social interactions topologies, provided that all agents have the same number of neighbors. In contrast to earlier findings in the literature on the transient nature of clustering, the inherent multiplicity of equilibria in the models of this paper allows for permanent effects of initial conditions. By exploring richer social settings, this paper goes beyond previous work. It also reinforces the arguments made by Brock and Durlauf on the importance of statistical mechanics models for the study of social interactions.

We examine the dynamics of social interaction with general topologies under the assumption that agents use the expectation of their neighbors' past decisions as the expectation of their neighbors' current decisions, which they consider as deterministic. We characterize fully existence and dynamic stability in the case of regular topologies and of random topologies as well. The dynamics of interdependent decisions depend critically on the assumption about expectations. The dynamics are stochastic and thus vastly more complicated if agents use their neighbors' actual past decisions as the expectation of their future decisions. The most general case of arbitrary social interactions topology may be studied as a Markov process, but because it is defined over a very large sample sample space, its high dimensionality makes it unwieldy. Nonetheless, it is associated with a stationary distribution. We characterize in detail the stochastic dynamics in such settings for the cases when interaction topologies are translation-invariant and interaction structures reversible. These conditions are satisfied in the cases of symmetric circular interaction and of global interaction, in particular. The stochastic dynamics of the Walrasian star interaction model do not satisfy these conditions but they may be handled directly. The stationary distribution can be characterized fully and coincides with the equilibrium distribution when agents make decisions with knowledge of their neighbors' actual decisions in a static setting. 


\section{$7 \quad$ References}

Anderson, T. W. (1971), The Statistical Analysis of Time Series, John Wiley and Sons, New York.

Azariadis, Costas (1993), Intertemporal Macroeconomics, Blackwell Publishers, Oxford.

Asavathiratham, Chalee (2000), "The Influence Model: A Tractable Representation for the Dynamics of Networked Markov Chains," Ph. D. thesis, Department of Electrical Engineering and Computer Science, MIT, October.

Asavathiratham, Chalee, Sandip Roy, Bernard Lesieutre, and George Verghese (2001), "The Influence Model," IEEE Control Systems Magazine, December, 52-64.

Bala, Venkatesh, and Sanjeev Goyal (1998), "Learning from Neighbours," Review of Economic Studies, 65, 595-621.

Bala, Venkatesh, and Sanjeev Goyal (2000), "An Noncooperative Model of Network Formation," Econometrica, 68, 5, 1181-1229.

Baxter, Rodney J. (1982), Exactly Solved Problems in Statistical Mechanics, Academic Press, New York.

Bigelis, Stephen, Emilio N. M. Cirillo, Joel L. Lebowitz, and Eugene R. Speer (1999), "Critical Droplets in Metastable States of Probabilistic Cellular Automata," Physical Review Letters E, 59, 4, April, 3935-3941.

Bisin, Alberto, Ulrich Horst, and Onur Özgür (2002), " Rational Expectations Equilibria of Economies with Local Interactions," working paper, New York University, Department of Economics, November.

Blume, Laurence E. (1997), "Population Games," in Arthur, W. Brian, Steven N. Durlauf and David Lane, Eds. (1997), The Economy as an Evolving Complex System II, Addison-Wesley, Menlo Park, CA., 425-460.

Blume, Laurence E., and Steven N. Durlauf (2002), "Equilibrium Concepts for Social Interaction Models," working paper, Department of Economics, University of Wisconsin, April.

Bramoullé, Yann (2001), "Complementarity and Social Networks," THEMA and Brookings working paper, October.

Brock, William A., and Steven N. Durlauf (2001), "Discrete Choice with Social Interactions," Review of Economic Studies, 68, 2, 235-260.

Brockwell, Peter J., and Richard A. Davis (1991), Time Series: Theory and Methods, second edition, Springer-Verlag, New York. 
Burt, Ronald S. (1980), "Models of Network Structure," Annual Reviews of Sociology, 6, 79-41.

Cass, David and Menahem Yaari (1966), "A Reexamination of The Pure Consumption-Loans Model," Journal of Political Economy, 74, 353-367.

Chen, Hsiao-Chi, James W. Friedman, and Jacques-Francois Thisse (1997), "Boundedly Rational Nash Equilibrium: Probabilistic Choice Approach," Games and Economic Behavior, 18, 3254 .

Courant, R. (1988), Differential and Integral Calculus, Vol. I, Wiley Interscience, New York.

Cox, D. R., and D. V. Hinkley (1974), Theoretical Statistics, Chapman and Hall, London.

Cvetković, Dragǒs M., Michael Doob, Ivan Gutman, and Aleksandar Torgasev (1988), Recent Results in the Theory of Graph Spectra, North-Holland, Amsterdam.

Cvetković, Dragǒs M., Michael Doob, and Horst Sachs (1995), Spectra of Graphs: Theory and Applications, Johann Ambrosius Barth Verlag, Heidelberg, 3rd edition.

Cvetković, Dragós M., and P. Rowlinson (1990), "The Largest Eigenvalue of A Graph: A Survey," Linear and Multilinear Algebra, 28, 3-33.

Davis, Philip J. (1979), Circulant Matrices, John Wiley and Sons, New York.

Durlauf, Steven N. (1997), "Statistical Mechanics Approaches to Socioeconomic Behavior," in Arthur, W. Brian, Steven N. Durlauf and David Lane, Eds. (1997), The Economy as an Evolving Complex System II, Addison-Wesley, Menlo Park, CA., 81-104.

Durrett, Richard, and Simon Levin (1994), "The Importance of Being Discrete (and Spatial)," Theoretical Population Biology, 46, 363-394.

Eisele, Theodor, and Richard S. Ellis (1983), "Symmetry Breaking and Random Waves for Magnetic Systems on a Circle," Zeitschrift für Wahrscheinlichkeitstheorie verwandte Gebiete, 63, 297-348.

Elkhader, A. S. (1992), "A Result on a Feedback System of Ordinary Differential Equations," Journal of Dynamics and Differential Equations, 4, 3, 399-418.

Ellis, Richard S. (1985), Entropy, Large Deviations, and Statistical Mechanics, Springer-Verlag, New York.

Ellison, Glenn (1993), "Learning, Local Interaction and Coordination," Econometrica, 61, 104771.

Epstein, Joshua M., and Robert Axtell (1996), Growing Artificial Societies, Brookings Institution Press/MIT Press. 
Erdös, Paul, and Alfred Renyi (1960), "On the Evolution of Random Graphs," Publications of the Mathematical Institute of the Hungarian Academy of Sciences, 5, 17-61.

Feller, William (1968), An Introduction to Probability Theory and Its Applications, Volume I, J. Wiley, New York.

Fujita, Masahisa, Paul M. Krugman, and Anthony J. Venables (1999), The Spatial Economy, MIT Press, Cambridge, MA.

Glaeser, Edward L., and José Scheinkman (2000), "Non-market Interactions," presented at the World Congress of the Econometric Society, Seattle, August.

Glaeser, Edward L., Bruce Sacerdote and José Scheinkman (1996), "Crime and Social Interactions," Quarterly Journal of Economics, 507-548.

Glauber, Roy J. (1963), "Time-Dependent Statistics of The Ising Model," Journal of Mathematical Physics, 4, 2, 294-307.

Griffeath, David (1976), "Introduction to Random Fields," Chapter 12, 425-458, in Kemeny, John G., J. Laurie Snell and Anthony W. Knapp, Denumerable Markov Chains, Springer-Verlag, New York.

Haag, Matthew, and Roger Lagunoff (2001), "Social Norms, Local Interaction and Neighborhood Planning," working paper, Department of Economics, Georgetown University, May.

Haller, Hans H., and Alexander V. Outkin (1999), "Best Response Dynamics and Neural Networks," working paper, Department of Economics, VPI\&SU, February.

Hirsch, Morris W. (1982), "Systems of Differential Equations which Are Competitive or Cooperative. I: Limit Sets," SIAM Journal of Mathematical Analysis, 13, 2, 167-179.

Horst, Ulrich, and Joseé A. Scheinkman (2003), "Equilibrium in Systems of Social Interactions," working paper, Princeton University, Department of Economics, February.

Goldberg, Samuel (1958), Introduction to Difference Equations, Wiley, New York.

Ioannides, Yannis M. (1997), "The Evolution of Trading Structures," in Arthur, W. Brian, Durlauf, Steven N., and David Lane, Eds. (1997), The Economy as an Evolving Complex System II, Addison-Wesley, Menlo Park, CA., 129-167.

Kehoe, Timothy J., and David K. Levine (1990), "The Economics of Indeterminacy in Overlapping Generations Models," Journal of Public Economics, 42, 219-243,

Kindermann, Ross, and J. Laurie Snell (1980), Markov Random Fields and Their Applications, American Mathematical Society, Providence, Rhode Island. 
Kirman, Alan P. (1993), "Whom or What Does the Representative Individual Represent?" Journal of Economic Perspectives, 6, 2, 117-136.

Kirman, Alan P. (1997), "The Economy as an Interactive System," in Arthur, W. Brian, Durlauf, Steven N., and David Lane, Eds. (1997), The Economy as an Evolving Complex System II, Addison-Wesley, Menlo Park, CA., 491-531.

Kooreman, Peter, and Adriaan R. Soetevent (2002), "A Discrete Choice Model with Social Interactions: An Analysis of High School Teen Behavior," University of Groningen, mimeo, February.

Mallet-Paret, John, and Hal L. Smith (1990), "The Poincaré-Bendixson Theorem for Monotone Cyclic Feedback Systems," Journal of Dynamics and Differential Equations, 2, 4, 367-421.

Manski, Charles F. (1997), "Identification of Anonymous Endogenous Social Interactions," in Arthur, W. Brian, Durlauf, Steven N., and David Lane, Eds. (1997), The Economy as an Evolving Complex System II, Addison-Wesley, Menlo Park, CA., 369-384.

McFadden, Daniel (1981), "Econometric Models of Probabilistic Choice," in Charles F. Manski and Daniel McFadden, ed., Structural Analysis of Discrete Data with Econometric Applications, Cambridge, Mass: MIT Press.

McKelvey, Richard D., and Thomas R. Palfrey (1995), "Quantal Response Equilibria for Normal Form Games," Games and Economic Behavior, 10, 6-38.

Morris, Stephen (2000), "Contagion," Review of Economic Studies, 76, 1, 57-78.

Padgett, John F. (1997), "The Emergence of Simple Ecologies of Skill: A Hypercycle Approach to Economic Organization," in Arthur, W. Brian, Durlauf, Steven N., and David Lane, Eds. (1997), The Economy as an Evolving Complex System II, Addison-Wesley, Menlo Park, CA., $199-221$.

Page, Scott E. (1997), "Network Structure Matters," University of Iowa, October, mimeo.

Preston, Christopher J., (1974), Gibbs States on Countable Sets, Cambridge University Press, Cambridge.

Puga, Diego, and Anthony J. Venables (1997), "Preferential Trading Arrangements and Industrial Location," Journal of International Economics, 3-4, 43, 347-368.

Quah, Danny T. (1999), "Cluster Emergence on a Global Continuum," London School of Economics, October.

Quah, Danny T. (2000), "Internet Cluster Emergence", European Economic Review, 44, 10321044. 
Roehner, Bertand M. (1995), Theory of Markets, Springer-Verlag, Berlin.

Schelling, Thomas C. (1978), Micromotives and Macrobehavior, Norton, New York.

Schmidt, Peter (1981), "Constraints on the Parameters in Simultaneous Tobit and Probit Models," p. 422-434, in Charles F. Manski and Daniel McFadden, ed., Structural Analysis of Discrete Data with Econometric Applications, Cambridge, Mass: MIT Press.

Soetevent, Adriaan R. (2003), "Equilibrium Properties of Finite Binary Choice Games," University of Groningen, mimeo, December.

Tamer, Elie (2002), "Empirical Strategies for Estimating Discrete Games with Multiple Equilibria," working paper, Princeton University, October.

Tamer, Elie (2003), "Incomplete Simultaneous Discrete Response Model with Multiple Equilibria," Review of Economic Studies, 70, 147-165.

Turing, Alan M. (1952), "The Chemical Basis of Morphogenesis," Philosophical Transactions of the Royal Society of London, 237, 37, 37-72.

Watts, Duncan J., and Steven H. Strogatz (1998), " Collective Dynamics of 'Small-World' Problems, Nature, 393, June 4, 440-442.

Young, H. Peyton (1993), "The Evolution of Conventions," Econometrica, 61, 57-84.

Young, H. Peyton (1998), Individual Strategy and Social Structure: An Evolutionary Theory of Institutions, Princeton University Press, Princeton. 


\section{Appendix: Proofs and Technical Material}

\subsection{Proof of Proposition 1.}

Proof of existence is guaranteed by Brower's fixed point theorem. It can be demonstrated graphically quite easily. Let the horizontal axis in Figure 2 denote $m_{1}$ and the vertical axis $m_{-1}$. Then Equ. (9) is plotted by the dashed curve and Equ. (10) by the full curve. The set of possibilities regarding the number and types of roots are obvious and summarized in the main text of the proposition above.

Q.E.D.

\subsection{Proof of Proposition 2.}

(a) Equ. (13) follows from (12) after setting $m=m_{i}$. The resulting solutions, designated as $m_{C I}^{*}$, are the spatial equivalent of a steady state, and it is for this reason that they are referred to as isotropic. Clearly, if $J_{B}=J_{F}=J$, then these solutions coincide with those for the mean field case. (b) This part of the proposition addresses the possibility that neighboring agents may not in the same state, at equilibrium. Let us set $m_{I}=\bar{m}_{I}$ and $m_{1}=\bar{m}_{1}$. By solving (12) for $m_{i+1}$ in terms of $\left(m_{i}, m_{i-1}\right)$, we have:

$$
m_{i+1}=\frac{1}{\frac{1}{2} \beta J_{F}}\left[\tanh ^{-1}\left(m_{i}\right)-\beta h\right]-\frac{J_{B}}{J_{F}} m_{i-1} .
$$

The mapping $\Theta$ defined above transforms the second-order spatial dependence expressed by the above equation into a first-order spatial dependence for a two-dimensional system, in terms of $\left(M_{i}^{1}, M_{i}^{2}\right)$, defined as $M_{i}^{1}=m_{i}$ and $M_{i}^{2}=m_{i-1}$. This mimics, of course, such transformations which are standard in the study of dynamical systems. It follows that

$$
\begin{aligned}
& M_{i+1}^{1}=\Theta^{1}\left(M_{i}^{1}, M_{i}^{2}\right) \\
& M_{i+1}^{2}=\Theta^{2}\left(M_{i}^{1}, M_{i}^{2}\right) .
\end{aligned}
$$

By iterating this mapping $I-1$ times, we see that $m_{1}$ and $m_{I}$ may be expressed as functions of $\bar{m}_{1}$ and $\bar{m}_{I}$. Therefore, if anisotropic equilibria exist, they would be the non-isotropic fixed points of the $I$ th iterate of the mapping $\Theta$.

(c).Working from (14) and (54), let $m_{1}=\bar{m}_{1}$ and $m_{3}=\bar{m}_{3}$. We define $\Theta_{[1]}, \Theta_{[2]}$, the first and second iterates of $\Theta$, and thus $m_{1}=\bar{m}_{1}$ and $m_{3}=\bar{m}_{3}$ as a fixed point. That is:

$$
\begin{gathered}
m_{2}=\Theta^{1}\left(\bar{m}_{1}, \bar{m}_{3}\right) \\
m_{1}=\Theta^{2}\left(\bar{m}_{1}, \bar{m}_{3}\right) \\
m_{3}=\Theta_{[1]}^{1}\left(\bar{m}_{1}, \bar{m}_{3}\right)=\frac{1}{\frac{1}{2} \beta J_{F}}\left[\tanh ^{-1}\left(\Theta^{1}\left(\bar{m}_{1}, \bar{m}_{3}\right)\right)-\beta h\right]-\frac{J_{B}}{J_{F}} \Theta^{2}\left(\bar{m}_{1}, \bar{m}_{3}\right), \\
m_{2}=\Theta_{[1]}^{2}\left(\bar{m}_{1}, \bar{m}_{3}\right)=\Theta^{1}\left(\bar{m}_{1}, \bar{m}_{3}\right), \\
m_{1}=\Theta_{[2]}^{1}\left(\bar{m}_{1}, \bar{m}_{3}\right)=\frac{1}{\frac{1}{2} \beta J_{F}}\left[\tanh ^{-1}\left(\Theta_{[1]}^{1}\left(\bar{m}_{1}, \bar{m}_{3}\right)\right)-\beta h\right]-\frac{J_{B}}{J_{F}} \Theta_{[1]}^{2}\left(\bar{m}_{1}, \bar{m}_{3}\right), \\
m_{3}=\Theta_{[2]}^{2}\left(\bar{m}_{1}, \bar{m}_{3}\right)=\Theta_{[1]}^{1}\left(\bar{m}_{1}, \bar{m}_{3}\right),
\end{gathered}
$$

Or,

$$
\tanh ^{-1}\left(\bar{m}_{1}\right)=\frac{1}{2} \beta J_{F} \tanh \left[\frac{1}{2} \beta J_{B} \bar{m}_{1}+\right.
$$




$$
\begin{gathered}
\left.\frac{1}{2} \beta J_{F} \tanh \left[\frac{1}{2} \beta J_{F} \bar{m}_{1}+\frac{J_{B}}{J_{F}}\left[\tanh ^{-1}\left(\bar{m}_{1}\right)-\beta h\right]-\frac{1}{2} \beta \frac{J_{B}^{2}}{J_{F}} \bar{m}_{3}+\beta h\right]+\beta h\right]+\frac{1}{2} \beta J_{B} \bar{m}_{3}+\beta h \\
\tanh ^{-1}\left(\bar{m}_{1}\right)=\frac{1}{2} \beta J_{B} \bar{m}_{3}+\frac{1}{2} \beta J_{F} \tanh \left[\frac{1}{2} \beta J_{B} \bar{m}_{1}+\frac{1}{2} \beta J_{F} \bar{m}_{3}+\beta h\right]+\beta h .
\end{gathered}
$$

Patient work shows that Equ. (58) and (59) admit the isotropic solution, and in addition have at least one solution.

Q.E.D.

\subsection{Proof of Proposition 3}

Consider $m_{C I}^{*}$, where $m_{C I}^{*}$ is one of the isotropic solutions of $(12), i=1, \ldots, I$, and linearize around to get a linear system of equations in terms of $\Delta m_{i}=m_{i}-m_{C I}^{*}, i=1, \ldots, I$. That is,

$$
J_{F} \Delta m_{i+1}-\frac{1}{\frac{1}{2} \beta \tau_{C}} \Delta m_{i}+J_{B} \Delta m_{i-1}, i=1, \ldots, I,
$$

where $\tau_{C} \equiv \tanh ^{\prime}\left(\beta h+\frac{1}{2} \beta\left(J_{B} m_{C I}^{*}+J_{F} m_{C I}^{*}\right)\right)$, which is nonzero. In addition, we have the "boundary condition" $\Delta m_{1}=\Delta m_{I+1}$.

According to Theorem 3.7, Goldberg (1958), the general solution of (60) may be written in terms of the roots of the characteristic equation

$$
J_{F} \zeta^{2}-\frac{1}{\frac{1}{2} \beta \tau_{C}} \zeta+J_{B}=0
$$

where $\zeta_{1}, \zeta_{2}$ may be conjugate complex. That is, the general solution is of the form $\Delta m_{i}=$ $C_{1} \zeta_{1}^{i}+C_{2} \zeta_{2}^{i}$, where $\zeta_{1}, \zeta_{2}$ are conjugate complex numbers and $C_{1}, C_{2}$ are constants. By substituting into the boundary condition we get the condition that $\zeta$ must be a $I$-th complex root of 1 . These two conditions are mutually inconsistent and we therefore conclude that there cannot be an anisotropic solution in the vicinity of an isotropic one, that is where the linearization of the nonlinear equations (12) is appropriate.

Q.E.D.

\subsection{Proof of Proposition 4}

Part (a). The proof is straightforward.

Part (b). With $L=1$, by inverting Equ. (12) and using Equ.'s (15) and (16), we may write for $m_{-1}$ and $m_{1}$ in Equ. (12) as functions of $m_{0}$. The examination of the multiplicity of the roots is similar to the case of mean field theory. See Figure 3.

Part (c). The mappings $\Theta_{L-1}^{+}(\cdot), \Theta_{L-1}^{-}(\cdot)$, are defined so as to express forward from the left end and backward from the right end the dependence among neighboring agents up until agents -1 and 1, respectively. For $\Theta_{L-1}^{+}$, by working with Equ. (12) for $i=-L+1$ in inverse form and by using (15), we have:

$$
m_{-L+2}=\frac{1}{\frac{1}{2} \beta J_{F}} \tanh ^{-1}\left(m_{-L+1}-2 \frac{\beta}{J_{F}} h\right)-\frac{J_{B}}{J_{F}} \tanh \left[\beta h+\beta J_{F} m_{-L+1}\right], i=1, \ldots, I,
$$

$m_{-L+2}$ as a function of $m_{-(L-1)}$, is well defined over its entire range. However, it is the inverse of this mapping, $m_{-(L-1)}=\mathcal{M}^{+}\left(m_{-(L-2)}\right)$, is well defined only over two disconnected intervals, $\left(-\infty, \theta_{-}\right)$ and $\left(\theta_{+}, \infty\right)$, over which it is monotonically increasing. Proceeding in this fashion, we apply Equ. 
(12) for $i=-L+2$ and use the definition of $\Theta^{1}$ to write $m_{-L+3}=\Theta^{1}\left(m_{-(L-2)}, m_{-(L-1)}\right)=$ $\Theta^{1}\left(m_{-L+2}, \mathcal{M}\left(m_{-L+2}\right)\right)$. Again, it is the inverse mapping, $m_{-L+2}=\vartheta^{+}\left(m_{-L+3}\right)$, that is necessary for expressing the forward recursion. And, for $-(L-4) \leq i \leq 1$, we iterate in this fashion forward until we get $m_{-1}$ as a function of $m_{0}$, which is referred to as $m_{-1}=\Theta_{L-1}^{+}\left(m_{0}\right)$.

For $\Theta_{L-1}^{-}$, we work in like manner but backwards from $i=L-1$ to $i=1$. Applying Equ. (12) for $i=L-1$ in inverse form, and by using (16), we may solve for $m_{L-2}$ as a function of $m_{L-1}$. Let the inverse of this mapping be defined by: $m_{L-1}=\mathcal{M}^{-}\left(m_{L-2}\right)$. From the definition of $\Theta_{1}$, we have that: $m_{L-3}=\Theta^{1}\left(m_{L-2}, m_{L-1}\right)$. Or:

$$
m_{L-3}=\Theta^{1}\left(m_{L-2}, \mathcal{M}^{-}\left(m_{L-2}\right)\right),
$$

from which we define the inverse mapping $m_{L-2}=\vartheta^{+}\left(m_{L-3}\right)$. And, for $1 \leq i \leq L-4$, we iterate in this fashion backward until we get $m_{1}$ as a function of $m_{0}$, which is referred to as $m_{1}=\Theta_{L-1}^{-}\left(m_{0}\right)$.

Finally, by applying Equ. (12) for $i=0$ yields (19). Working graphically, and under the assumption that $\beta>0[\beta<0]$ a solution for $m_{0}^{*}$ exists, lies in $((0,1))[(-1,0)]$, and is single. We note that this solution is different from the isotropic one, as the transformations we employ would not hold. It follows by construction that the $m_{i}^{*}$ s differ from one another. Again, if $J_{B}=J_{F}$, then the solution is symmetric relative to $i=0$.

Q.E.D.

\subsection{Proof of Proposition 5}

By substituting in (12), $i=L-1$, for $m_{L}$, from Equ. (15), and in (12), $i=-L+1$, for $m_{-L}$ from (16), we have, respectively, for $i=L-1$, and for $i=-L+1$,

$$
\begin{aligned}
m_{L-1} & \left.=\tanh \left[\beta h+\frac{1}{2} \beta J_{B} m_{L-2}+\frac{1}{2} \beta J_{F} \tanh \left[\beta h+\beta J_{B} m_{L-1}\right]\right)\right] ; \\
m_{-L+1} & \left.=\tanh \left[\beta h+\frac{1}{2} \beta J_{F} m_{-L+2}+\frac{1}{2} \beta J_{B} \tanh \left[\beta h+\beta J_{F} m_{-L+1}\right]\right)\right] .
\end{aligned}
$$

These equations along with $(12),-L+2 \leq i \leq L-2$, are $2 L-1$ nonlinear equations in the $2 L-1$ unknowns. Consider $m_{\ell}^{*}$, one of the isotropic solutions of $(12), i=-L+2, \ldots, L-2$, and $m_{-\ell}^{*}$, $m_{+\ell}^{*}$, the associated solutions from (15) and (16), and linearize around them to get a linear system of equations in terms of the deviations $\Delta m_{i}$, of the anisotropic solution from the isotropic one, for $-L+2 \leq i \leq L-2$. The corresponding equations for $\Delta m_{-L+1}$ and for $\Delta m_{L-1}$ are more complicated but we ignore that complication by assuming that $m_{-L+1}$ and $m_{L-1}$ are fixed. Therefore,

$$
\Delta m_{-L+1}=0, \Delta m_{L-1}=0 .
$$

The remaining deviations satisfy Equ. (60), for $-L+2 \leq i \leq L-2$, where $\tau^{L} \equiv \tanh ^{\prime}\left(\beta h+\frac{1}{2} \beta\left(J_{B} m_{\ell}^{*}+J_{F} m_{\ell}^{*}\right)\right)$, which is nonzero. This together with the boundary conditions may be analyzed further by following ( in fact, replicating ) the analysis in Goldberg (1958), p. 184-188 to show that anisotropic solutions exist for only certain specific values of the coefficients in Equ. (60). There is only a finite number of those values, and therefore we conclude that, in general, there are no anisotropic solution in the vicinity of one of the isotropic solutions.

Q.E.D. 


\subsection{Derivation of Equ. (27).}

To solve for Prob $\left\{\omega_{1}\right\}$ next, we work with the probability of the event $\left\{\omega_{1} \cdot\left\{\sum_{i=2}^{i=I} \omega_{i}=k\right\}\right\}$. By summing over all possible values of $k$, we obtain an expression for the probability Prob $\left\{\omega_{1}\right\}$. That is, the state of individual 1 must be consistent with all possible realizations of the states of her neighbors $i=2, \ldots, I$ :

$$
\operatorname{Prob}\left\{\omega_{1}=1\right\}=\sum_{k} \operatorname{Prob}\left\{\omega_{1}=1 \mid \sum_{i=2}^{I} \omega_{i}=k\right\} \cdot \operatorname{Prob}\left\{\sum_{i=2}^{I} \omega_{i}=k\right\} .
$$

The first term of the summand in the RHS above is given from (25). The second term may be obtained as

$$
\sum_{\omega_{1}=1,-1} \operatorname{Prob}\left\{\omega_{1}\right\} \cdot \operatorname{Prob}\left\{\sum_{i=2}^{I} \omega_{i}=k \mid \omega_{1}\right\},
$$

by summing over all values of $\omega_{1}$. That is, the state of the agents on the periphery must be consistent with all possible realizations of the state of the central agent:

$$
\begin{gathered}
\operatorname{Prob}\left\{\sum_{i=2}^{I} \omega_{i}=k\right\} \\
=\operatorname{Prob}\left\{\omega_{1}=1\right\} \cdot \operatorname{Prob}\left\{\sum_{i=2}^{I} \omega_{i}=k \mid \omega_{1}=1\right\}+\left[1-\operatorname{Prob}\left\{\omega_{1}=1\right\}\right] \cdot \operatorname{Prob}\left\{\sum_{i=2}^{I} \omega_{i}=k \mid \omega_{1}=-1\right\} .
\end{gathered}
$$

The probability distributions for the random variables $\left\{\sum_{i=2}^{I} \omega_{i} \mid \omega_{1}=1\right\}$ and $\left\{\sum_{i=2}^{I} \omega_{i} \mid \omega_{1}=-1\right\}$ are given from the binomial distribution and thus obtained by means of the formula for $\frac{1}{2}(I+k-1)$ successes in $I-1$ independent Bernoulli trials. Therefore, by substituting from (62) into the RHS of (61) we may solve the resulting linear equation for Prob $\left\{\omega_{1}=1\right\}$ and obtain Equ. (27).

Q.E.D.

\subsection{Derivations for the Cyclical Interaction Model as One-Dimensional Nearest Neighbor Ising Model}

For the cyclical interaction model, the probability of a social state $\tilde{\omega}$ is given by

$$
\Pi(\tilde{\omega})=\frac{1}{\overline{\mathcal{D}}} \exp \left[\sum_{\kappa \subset V} \mathcal{D}_{\kappa}(\tilde{\omega})\right]
$$

, with $\mathcal{D}$ defined as

$$
\mathcal{D}(\tilde{\omega}) \equiv \beta J \sum_{j=1}^{I} \omega_{j} \omega_{j+1}+\beta h \sum_{j=1}^{I} \omega_{i},
$$

and $\overline{\mathcal{D}}$, the partition function defined as

$$
\overline{\mathcal{D}}=\sum_{\widetilde{\omega}} \exp \left[\beta J \sum_{j=1}^{I} \omega_{j} \omega_{j+1}+\beta h \sum_{j=1}^{I} \omega_{j}\right],
$$

where summation over $\tilde{\omega}$ is understood over all $2^{I}$ possible realizations of the social state $\tilde{\omega}$. Following the classic treatment of Baxter (1982), p. 32-36, we may work as follows. 
The exponential function in (65) may be factored into terms each of them being symmetric and involving only neighboring states,

$$
V\left(\omega, \omega^{\prime}\right) \equiv \exp \left[\beta J \omega \omega^{\prime}+\frac{1}{2} \beta h\left(\omega+\omega^{\prime}\right)\right]
$$

thus yielding

$$
\overline{\mathcal{D}}=\sum_{\tilde{\omega}} V\left(\omega_{1}, \omega_{2}\right) \cdot V\left(\omega_{2}, \omega_{3}\right) \cdot \ldots \cdot V\left(\omega_{I-1}, \omega_{I}\right) \cdot V\left(\omega_{I}, \omega_{1}\right)
$$

From the definition of $V\left(\omega, \omega^{\prime}\right)$, we may write the $2 \times 2$ matrix with elements all possible values of $V\left(\omega, \omega^{\prime}\right)$,

$$
\mathbf{V}=\left[\begin{array}{cc}
e^{\beta J+\beta h} & e^{-\beta J} \\
e^{-\beta J} & e^{\beta J-\beta h}
\end{array}\right] .
$$

Then the summation over $\omega_{2}, \ldots, \omega_{I}$ in (66) can be regarded as successive matrix multiplications and the summation over $\omega_{1}$ as the taking of a trace, so that $\overline{\mathcal{D}}=\operatorname{Trace}\left\{\mathbf{V}^{I}\right\}$. It follows that:

$$
\overline{\mathcal{D}}=\text { Trace }\left\{\mathbf{P}\left[\begin{array}{cc}
\lambda_{1}^{I} & 0 \\
0 & \lambda_{2}^{I}
\end{array}\right] \mathbf{P}^{-1}\right\}=\lambda_{1}^{I}+\lambda_{2}^{I},
$$

where $\lambda_{1}, \lambda_{2}, \lambda_{1}>\lambda_{2}$, are the two distinct real eigenvalues of $\mathbf{V}$, a positive symmetric matrix. It follows from the characteristic equation of $\mathbf{V}$ that the eigenvalues are either both positive, if $J>0$, or one is positive (the absolutely larger of them) and the other is negative, if $J<0$. Therefore, $\overline{\mathcal{D}}$ is always positive.

From (63), the probability of any social state $\tilde{\omega}$ is given by

$$
\operatorname{Prob}(\tilde{\omega}))=\frac{\exp [\mathcal{D}(\tilde{\omega})]}{\operatorname{Trace}\left\{\mathbf{V}^{I}\right\}}
$$

We may obtain expressions for the first, second as well as other moments of the elements of $\tilde{\omega}$. In particular, the mean state of an agent is:

$$
\mathcal{E}\left[\omega_{i}\right]=\frac{\operatorname{Trace}\left\{\mathbf{S V}^{I}\right\}}{\operatorname{Trace}\left\{\mathbf{V}^{I}\right\}} ;
$$

the generalized second moment is:

$$
\mathcal{E}\left[\omega_{i} \omega_{j}\right]=\frac{\operatorname{Trace}\left\{\mathbf{S V}^{j-i} \mathbf{S} \mathbf{V}^{I+i-j}\right\}}{\operatorname{Trace}\left\{\mathbf{V}^{I}\right\}}, 0 \leq j-i \leq I,
$$

where $\mathrm{S}=\left(\begin{array}{cc}1 & 0 \\ 0 & -1\end{array}\right)$. The marginal probability for the state of each agent is given in Equ. (22), where the auxiliary variable $\xi$ is defined implicitly by:

$$
\cot 2 \xi=e^{\beta J} \frac{e^{\beta h}-e^{-\beta h}}{2} \equiv e^{\beta J} \sinh (\beta h), 0<\xi<\frac{\pi}{2} .
$$

Just as in the case of the Walrasian star model, the marginal probability describing the state of each agent is uniquely defined in terms of the fundamentals.

When $I \rightarrow \infty$, while $j-i$ remains finite, Baxter, op. cit. shows that the mean decision of each agent is given by (23) and then product of any two decisions by (24). 


\subsection{Analytics of the Dynamics of the Circular Interaction Model}

We have in closed form, from Brockwell and Davis (1992), p. 133-138, and Anderson (1971), Theorem 6.5.2, p. 279-281, the eigenvalues and eigenvectors of $\frac{1}{2}$ times the adjacency matrix for the cyclical interaction, $\boldsymbol{\Gamma}_{C}$. Specifically, if $I$ is odd, the eigenvalues of this matrix are

$$
\left\{\cos 0=1, \cos \left(\frac{2 \pi}{I}\right), \cos \left(\frac{2 \pi}{I}\right), \cos \left(\frac{4 \pi}{I}\right), \cos \left(\frac{4 \pi}{I}\right), \ldots, \cos \left(\frac{(I-1) \pi}{I}\right), \cos \left(\frac{(I-1) \pi}{I}\right)\right\} .
$$

The eigenvalues come in pairs, so that there are $1+\frac{I-1}{2}$ distinct roots. The eigenvector corresponding to the eigenvalue 1 is $(1,1, \ldots, 1)^{\mathrm{T}}$, and to the eigenvalues $\cos \frac{j 2 \pi}{T}, j \neq 0, \frac{I}{2}$, there correspond the eigenvectors

$$
\begin{aligned}
& \left(\cos \left(j \frac{2 \pi}{I}\right), \cos \left(j \frac{4 \pi}{I}\right), \ldots, 1\right)^{\mathrm{T}}, \\
& \left(\sin \left(j \frac{2 \pi}{I}\right), \sin \left(j \frac{4 \pi}{I}\right), \ldots, 0\right)^{\mathrm{T}},
\end{aligned}
$$

if $I$ is odd. If $I$ is even, the eigenvalues are

$$
\left\{\cos 0=1, \cos \left(\frac{2 \pi}{I}\right), \cos \left(\frac{2 \pi}{I}\right), \cos \left(\frac{4 \pi}{I}\right), \cos \left(\frac{4 \pi}{I}\right), \ldots, \cos \left(\frac{(I-2) \pi}{I}\right), \cos \left(\frac{(I-2) \pi}{I}, \cos \pi=-1\right)\right\} .
$$

The eigenvector corresponding to the eigenvalue 1 is $(1,1, \ldots, 1)^{\mathrm{T}}$, and to the eigenvalues $\cos \frac{j 2 \pi}{T}, j \neq$ $0, \frac{I}{2}$, the same as above. The eigenvector corresponding to the eigenvalue -1 , is $(-1,1,-1, \ldots, 1)^{\mathrm{T}}$.

Instead of this approach, we may work, following Turing (1952) and Glauber $(1963)^{19}$, from first principles and seek a general solution for Equ. (46) in the form $A \zeta^{i} \rho^{t}$, where $A$ is a constant and $\rho, \zeta$ are unknown, generally complex, numbers to be determined. Then we substitute into Equ. (46) to find its general solution for the special case of $J_{B}=J_{F}=J$. We note that for cyclical symmetry, it must be the case that $\zeta^{I}=1$. In other words, the complex number $\zeta$ must assume the values of the $I$ basic complex roots of 1 , that is: $\left(1, \exp \left[\frac{2 \pi}{I} \sqrt{-1}\right], \ldots, \exp \left[\frac{2 \pi(I-1)}{I} \sqrt{-1}\right]\right)$. We then substitute into Equ. (46) and obtain the eigenvalues: $\rho=\tau^{C} J \zeta^{-1}+\tau^{C} J \zeta$, where $\tau^{C}:=$ $\tanh ^{\prime}\left(\beta h+\frac{1}{2} \beta\left(J m_{i-1}^{*}+J m_{i+1}^{*}\right)\right)$. Proceeding in this fashion is rather tedious, however.

This solution technique was employed by Turing (1952), and was adapted by Fujita, Krugman and Venables (1999), p. 85-95, in a continuous space and time model of product differentiation, by introducing finite Fourier transforms. A special case of Glauber's model, the "infinite ring" case, that is when $I \rightarrow \infty$, is solved by Ellis (1985), Theorem V. 10.4, p. 190-203. The circle model, studied in depth by Eisele and Ellis (1983), gives rise to some features which are absent from the Curie-Weiss model, namely a new kind of phase transition described in terms of random waves. Roehner (1995), p. 353-355 presents dynamic random fields on continuous spherical surfaces.

The general properties of the dynamics of differential equations of this type have received attention in the literature. Notable are the contributions of Hirsch, who studies differential equations whose Jacobian matrices have off-diagonal terms that are either non-positive (competitive) or nonnegative (cooperative). Hirsch (1982), in particular, shows that such systems of ordinary differential equations have bounded solutions that converge to steady states or to periodic orbits. Mallet-Paret and Smith (1990) allow only backward feedback and show that the Poincaré-Bendixson theorem

\footnotetext{
${ }^{19} \mathrm{I}$ am grateful to Bertrand Roehner for bringing Glauber (1963) to my attention. Glauber works with the master equation, a version of the Chapman-Kolmogorov equation in continuous time. This particular use of the ChapmanKolmogorov equation has become known as "Glauber dynamics" in the neural networks literature.
} 
holds, roughly speaking, for such equations. Elkhader (1992) is the only paper that allows for backward and forward feedback. His model is general but slightly different from Equ. (40), in that it lacks complete cyclical symmetry.

\subsection{Dynamics with Path Interactions}

By linearizing respectively for agents $-L,-(L-1), \ldots, L-1$, and $L$,

$$
\begin{gathered}
\left.\tau^{-L}:=\tanh ^{\prime}\left(\beta h+\beta J_{F} m_{-(L-1)}^{*}\right)\right), \\
\tau^{L}:=\tanh ^{\prime}\left(\beta h+\frac{1}{2} \beta\left(J_{B} m_{i-1}^{*}+J_{F} m_{i+1}^{*}\right)\right), \\
\left.\tau^{+L}:=\tanh ^{\prime}\left(\beta h+\beta J_{B} m_{L-1}^{*}\right)\right) .
\end{gathered}
$$

For the symmetric case where $J_{F}=J_{B}=J$, and given that we linearize around an isotropic equilibrium, we have that: $\tau^{-L}=\tau^{+L}=\tau^{L}$. We express Equ. (41) - Equ. (43) as a system of $2 L+1$ equations in matrix form:

$$
\left[\begin{array}{c}
\Delta m_{-L}(t) \\
\cdot \\
\Delta m_{0}(t) \\
\cdot \\
\Delta m_{L}(t)
\end{array}\right]=\beta J \tau^{L} \frac{1}{2}\left[\begin{array}{ccccccc}
0 & 1 & 0 & . & 0 & 0 & 0 \\
1 & 0 & 1 & . & 0 & 0 & 0 \\
\cdot & . & . & . & . & . & \cdot \\
0 & 0 & 0 & \cdot & 1 & 0 & 1 \\
0 & 0 & 0 & . & 0 & 1 & 0
\end{array}\right]\left[\begin{array}{c}
\Delta m_{-L}(t-1) \\
\dot{\Delta} m_{0}(t-1) \\
\dot{\Delta} \\
\Delta m_{L}(t-1)
\end{array}\right] .
$$

The matrix in the r.h.s. of (71) is no longer a circulant. However, the eigenvalues and eigenvectors of $\frac{1}{2}$ times the adjacency, the matrix in the R.H.S. of (71) have been studied by Anderson, op. cit., p. 290, Equ. (62). The adjacency matrix is an $(2 I+1) \times(2 I+1)$ matrix, whose first and last rows are $(0,1, \ldots, 0)$ and $(0,0, \ldots, 1,0)$ respectively and rows 2 through $2 I$ are the $2 I+1$ vector $(1,0,1, \ldots, 0)$ and its permutations. Its eigenvalues are given by: $2 \cos \left(\frac{\pi}{2(I+1)} i\right), i=1, \ldots, 2 I+1$; the corresponding eigenvectors are given by

$$
\left(\sin \left(\frac{\pi}{2} \frac{s}{L+1}\right), \sin \left(\frac{\pi}{2} \frac{2 s}{L+1}\right), \ldots, \sin \left(\frac{\pi}{2} \frac{s(2 L+1)}{L+1}\right)\right)^{\mathrm{T}}, s=1, \ldots, 2 L+1 .
$$

This result allows us to solve Equ. (71) in terms of the eigenvectors, just like (47) and obtain Equ. (50) in the main text.

\subsection{Dynamics with Path Interactions with $z$ Transforms when $L \rightarrow \infty$}

It is convenient to apply the $z$-transform to the entire system of equations (42). This is the discretetime counterpart of the treatment in continuous time by Glauber, op. cit., for the linear ring case. We define $\mathcal{L}(z, t)$, the $z$-transform of the sequence of deviations $\mathcal{D} M(t)=\left\{\Delta m_{i}(t)\right\}_{i=-\infty}^{i=\infty}$ :

$$
\mathcal{L}(z, t)=\sum_{i=-\infty}^{i=\infty} z^{i} \Delta m_{i}(t)
$$

It follows that by multiplying both sides of (46) by $z^{i}$ and summing up for all $i$ 's, we have:

$$
\mathcal{L}(z, t)=\frac{1}{2} \beta J \tau^{L}\left(z+z^{-1}\right) \mathcal{L}(z, t-1)
$$


This may be solved to yield

$$
\mathcal{L}(z, t)=\mathcal{L}(z, 0)\left(\frac{1}{2} \beta J \tau^{L}\right)^{t} z^{-t}\left(z^{2}+1\right)^{t}
$$

$\mathcal{L}(z, t)$ may be obtained as power series by noticing that the term $\left(z^{2}+1\right)^{t}$ may be written in terms of the binomial expansion formula.

Intuitively, $\mathcal{L}(z, 0)$ carries the impact of initial conditions. We may solve for $\mathcal{L}(z, t)$ by assuming a set of initial conditions. Suppose, for example, that all deviations at 0 are equal to 0 except for $\Delta_{0}(0)=1$. In that case, $\mathcal{L}(z, 0)=1$, and

$$
\mathcal{L}(z, t)=\left(\frac{1}{2} \beta J \tau^{L}\right)^{t} \sum_{k=0}^{t} \frac{t !}{k !(t-k) !} z^{2 k-t} .
$$

The solutions for $\Delta_{i}(t)$ may be recovered from $\mathcal{L}(z, t)$ in the obvious way, as the coefficients of the powers of the $z^{i}$ 's. That is, the solution for $\Delta m_{i}(t)$ is given by the coefficient of $z^{i}$ in the power expansion for $\mathcal{L}(z, t)$. Writing the terms of the summation in the r.h.s. of (A.1) above yields:

$$
z^{-t}+t z^{2-t}+\frac{(t-1) t}{2} z^{4-t}+\frac{(t-2)(t-1) t}{6} z^{6-t}+\ldots+t z^{t-2}+z^{t} .
$$

Since for every $t$, the binomial coefficient in the r.h.s. of (A.1) increases with $k$, reaches a maximum, and then declines, the impact on the coefficients of the powers of $z$ depends on the magnitude of $\left(\frac{1}{2} \beta J \tau^{L}\right)^{t}$, as well, which may be increasing or decreasing over time, depending upon the which particular isotropic equilibrium we start from.

\subsection{Proof of Proposition 7}

Part I.

(a) This part follows from the discussion of subsection 2.1.1. That they are all isotropic follows from symmetry. To see this, assume that for agent $i$, her neighbors are at nonisotropic steady states. By iterating forward with respect to $i$ we reach a contradiction. Therefore, when Equ. (51) is taken at a steady state, $\boldsymbol{\Gamma}_{i} \mathbf{m}=d m_{i}$ and $m_{i}=m^{*}$, where $m^{*}$ is a solution of Equ. (5) is a solution. It is easy to see that it is the only solution.

(b) This part is also obvious after we linearize around a uniform steady state and apply matrix notation.

(c) Since the adjacency matrix $\boldsymbol{\Gamma}$ is symmetric and positive, it has real eigenvalues and a non-negative maximal eigenvalue, whose magnitude absolutely exceeds all other eigenvalues. This eigenvalue is "squeezed" between the average degree of $G$ and is maximal degree, which in our case is equal to $I-1$ [ Cvetkovic, et al. (1995), 381-382 ]. However, all agents have the same number of neighbors, therefore, the maximal eigenvalue is equal to $d$.

When the economy is at a steady state with all $m_{i}$ 's assuming either one of the asymmetric values $m_{-}^{*}, m_{+}^{*}$, or the symmetric value $m^{*}$, as defined in subsection 2.1.1 above, if they exist, then $\tau$ simplifies to become equal to $\tanh ^{\prime}[\beta h+\beta J m]$. Condition (53) follows as a necessary and sufficient condition for the solution of (52) to be stable (unstable). It would be stable if $m_{i}=m_{-}^{*}, m_{+}^{*}$, and unstable if $m_{i}=m^{*}, \forall i$.

Part II. The proof readily follows from Brower's fixed point theorem. The mapping from $[-1,1]^{I}$ into itself, defined by (51) for $\mathbf{m}_{t}=\mathbf{m}$, has at least one fixed point. 
Part III. The proof follows from the fact that the row elements of the matrix consisting of rows $\frac{1}{\nu(i) \mid} \boldsymbol{\Gamma}_{i}$ are positive and sum up to 1 . Therefore, it is a stochastic matrix, whose maximal eigenvalue is equal to 1. Condition (53) holds, provided that its l.h.s. is evaluated at the appropriate steady state, at least one of which exists, by Part 2 .

Q.E.D. 